Preprint typeset in JINST style - HYPER VERSION

LAL 10-44

\title{
Per Mill Level Control of the Circular Polarisation of the Laser Beam for a Fabry-Perot Cavity Polarimeter at HERA
}

\author{
V. Brisson, R. Chiche, M. Jacquet, C. Pascaud, V. Soskov, Z. Zhang, F. Zomer \\ Laboratoire de l'Accélérateur Linéaire, Univ. Paris-Sud et IN2P3/CNRS, \\ Orsay, France \\ E-mail: mjacquetelal.in2p3.fr
}

M. Beckingham, N. Coppola

DESY

Hamburg, Germany

\begin{abstract}
A precise and fast Fabry-Perot cavity polarimeter, installed in the HERA tunnel in the summer of 2003, was used to measure the longitudinal polarisation of the lepton beam. A complete theoretical model has been developed in order to control at the per mill level the degree of circular polarisation of the laser beam. The transport of this quantity within the whole optical setup has also been performed and controlled at the same level of precision. This is the first time that such a precision is achieved in the difficult, hostile and noisy environment of a particle collider.
\end{abstract}

KEYWORDS: Particle collider, Polarimeter, Ellipsometer, Light polarisation, Quarter Wave Plate.

${ }^{*}$ Corresponding author.

${ }^{\dagger}$ Now at Albert-Ludwigs-University Freiburg, Freiburg, Germany.

\#Now at European XFEL GmbH, Hamburg, Germany. 


\section{Contents}

1. Introduction 1

2. Ellipsometer characterisation 2

2.1 General optical setup and ellipsometer components

2.1.1 General optical setup B

2.1.2 Ellipsometer components

2.2 Model of the ellipsometer and $\chi^{2}$ function

2.2.1 Model of the ellipsometer 6

2.2.2 $\chi^{2}$ function 7

2.3 Ellipsometer parameter determination 8

2.3.1 The data calibration samples 8

2.3.2 Correlation between $e_{\mathrm{QWP}}$ and $S_{3}$

2.3 .3 Results 10

3. Regular measurements of $S_{3}$ and systematics studies 10

3.1 Measurements and systematic uncertainty from the ellipsometer 10

3.2 Transport of $S_{3}$ from ellipsometer to cavity center 12

3.2.1 Parasitic ellipticity from cavity center to ellipsometer entrance 12

3.2.2 Exit transfer matrix $M_{T}$

$3.2 .3 \quad S_{3}$ at the exit of the cavity 13

3.3 Overall $S_{3}$ uncertainty 14

๑. Coherence of $S_{3}$ along the whole optical system 14

4.1 Determination of $M_{E}$

4.2 Coherence of $S_{3}$

5. Summary 17

\section{Introduction}

In a companion article [1] we describe a Compton polarimeter installed at HERA using a FabryPerot resonator to enhance the laser beam power. A relative statistical precision of $2 \%$ per bunch and per minute was achieved on the longitudinal polarisation $P_{z}$ measurement of the electron beam with an estimated relative systematic uncertainty of about $1 \%$. One of the systematic error sources is related to the determination of the degree of circular polarisation $S_{3}$ of the laser beam at the electron-laser interaction point (IP). Since only the product $S_{3} P_{z}$ is determined in Compton polarimetry (see Eq.(5) in [1]), we have to measure precisely $S_{3}$ in order to achieve the same level 
of precision for $P_{z}$. The uncertainty on the $S_{3}$ determination comes mainly from two sources: (1) the measurement of $S_{3}$ itself performed with an ellipsometer usually located close to, but outside, the electron beam pipe and, (2) the transport of the measured $S_{3}$ value through optical elements and vacuum window up to the electron-laser IP. The purpose of the present article is to describe the experimental setup and methods that we have used to reach a few per mill level of systematic uncertainty on $S_{3}$.

The key component of the experimental setup [1] is an ellipsometer similar to those of the SLAC [2, 3] and Jlab [4, 5] Compton polarimeters. It is composed of a quarter wave plate (QWP), a linear polariser and various photo-detectors. Since an optical model is needed to reconstruct $S_{3}$ from the photometric measurements performed after the polariser, the model accuracy has to be controlled below the per mill level. The QWP is a crucial component of the ellipsometer. It is usually anti-reflection coated with double layers and thus taken as a simple delay plate in basic optical models [6]. However, the reflectance of such coated plates is typically of the order of $0.5 \%$, thus limiting the model accuracy to the same level. In order to decrease the model uncertainty, we followed the work of [ [7] by choosing an uncoated quartz QWP of high optical quality. In doing so we have to account for multiple reflections inside the anisotropic uniaxial QWP, to model the plate defects and the experimental misalignments, and to perform a fine calibration of the plate thickness. The implementation of an uncoated QWP in the ellipsometer together with a thorough investigation of theoretical models and detector effects within an accelerator environment has never been reported previously and is one of the main topics of this article.

In order to control the transport of accurately measured $S_{3}$ up to the IP, we follow the method developed for the polarimeter at SLAC [8] by modeling the optical elements located between the IP and the ellipsometer, and use optical theorems demonstrated by Jones [9]. In an accelerator this transport is an important issue since optical elements are always present between the IP and the ellipsometer. All birefringence biases from these elements are therefore studied and/or modeled to conserve the required precision on $S_{3}$ at the IP. We also perform a polarisation transport study from the IP up to the laser head by using the optical theorems in [10]. The implementation of these methods is the second topic of this article. To our knowledge they have not been applied in the context of accelerators to the level of accuracy presented here.

The ellipsometer and the characterisation of its optical components are described in Sect. 2. In Sect. 3, the determination of $S_{3}$ with the ellipsometer and the transport from the ellipsometer to the electron-laser IP are described. Finally, in Sect. 4 , the characterisation of the entrance optical line is performed in order to study the coherence of $S_{3}$ along the whole optical system and in particular the coherence of $S_{3}$ at the entrance and at the exit of the Fabry-Perot cavity.

\section{Ellipsometer characterisation}

In this section, the general optical setup and the characterisation of the ellipsometer are described. The principle of an ellipsometer is to send a light beam, of any unknown polarisation, through a QWP. By rotating the plate, the polarisation state of the light is modified and the state at the exit of the plate depends on the state at the entrance. A polariser (Wollaston prism) placed behind the plate spatially separates the beam into two orthogonal linearly polarised states. The analysis of the 
intensities of these two beams in photo-detectors, for various azimuthal angles of the QWP, allows the deduction of the polarisation of the incident beam.

\subsection{General optical setup and ellipsometer components}

\subsubsection{General optical setup}

A schematic overview of the Fabry-Perot cavity optical setup is presented in Fig. 1. A Nd:YAG laser beam of $1064 \mathrm{~nm}$ wavelength passes first through a Glan-Thomson prism in order to provide a purely linearly polarised state and then through an entrance QWP, noted QWP ent This is mounted at the center of a motorised rotating stage to adjust the azimuthal angle $\phi_{\mathrm{ent}}$ and thus to provide an elliptical polarised state. The Glan allows also the beam to pass and to go back after its reflection by the cavity entrance mirror. The reflected beam is analysed in a photodiode, $\mathrm{pd}_{\mathrm{ent}}$, first to determine the azimuthal angles of the plate $\mathrm{QWP}_{\text {ent }}$ for which the light is circularly polarised and secondly to conduct a study on the entrance optical line (Sect. 4.1). The beam then passes through the entrance optics which is composed of a glass plate and two lenses. The glass plate is used to pick up a fraction of the beam for the locking procedure exploiting the "Pound-Drever" technique [11], and the lenses are used to match the laser beam to the cavity fundamental mode. The beam is then precisely aligned with four mirrors (of which two are motorised) before entering a two meter long cavity.

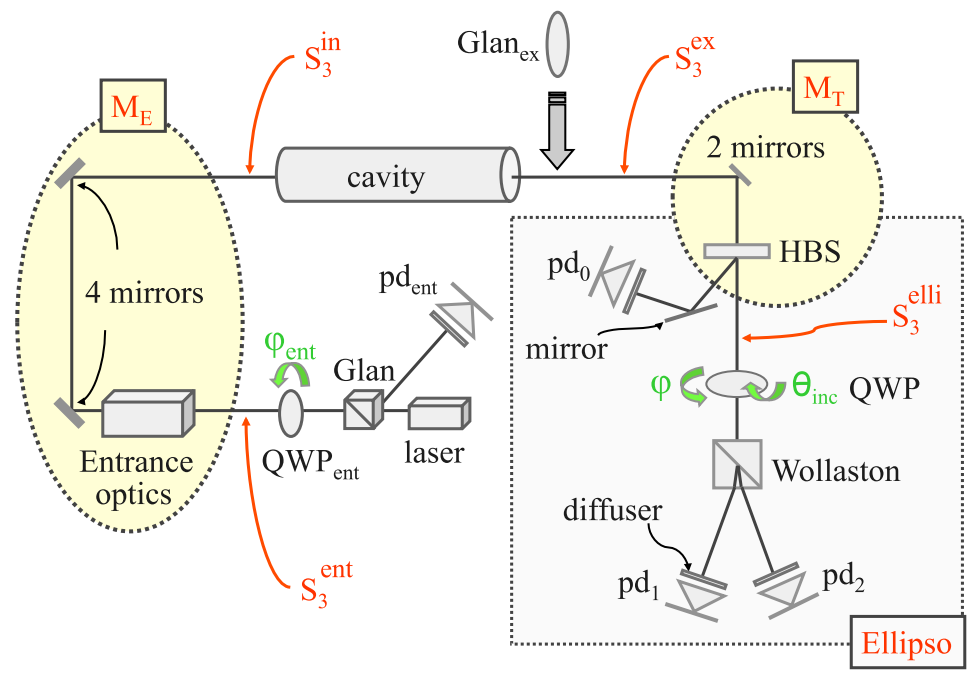

Figure 1. A schematic view of the Fabry-Perot cavity optical system installed in the HERA tunnel. The box "entrance optics" is composed of a glass plate and two lenses. The location of four determination points

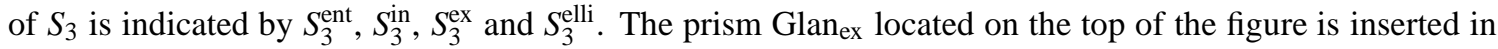
the optical line only for a dedicated study described in Sect. 3.2.2. $M_{E}$ and $M_{T}$ are two transfer matrices discussed in Sects. 3 and 4 .

At the exit of the cavity, the beam is guided with two mirrors to enter the ellipsometer, labeled "Ellipso", which is mainly composed of a QWP followed by a Wollaston prism. The beam passes first through a holographic beam sampler (HBS) in order to extract a small fraction of the entrance 
power (about $1 \%$ of the incident beam). This fraction of the beam is measured in photodiode $\mathrm{pd}_{0}$ and is used as a reference intensity to compensate the effects due to possible laser power variations. The main beam emerging from the HBS enters the QWP. The QWP is mounted at the center of a motorised rotating stage to adjust the azimuthal angle $\phi$. Two screws allow the alignment between the plate and the rotating stage to be adjusted. The rotating stage itself is mounted on a two-axis horizontal stage, a vertical translation stage and an angle-tilting stage to position the beam impact point at the mount center of the rotating stage and to adjust the incident angle $\theta_{\text {inc }}$ between the laser beam and the plate normal direction. The linear and tilted stages are manually controlled with micrometric screws. The Wollaston prism separates the laser beam spatially into two linearly and orthogonally polarised components, and the two transmitted beams from the Wollaston are detected in photodiodes $\mathrm{pd}_{1}$ and $\mathrm{pd}_{2}$. Diffusers are placed in front of each photodiode in order to adjust the power entering the photodiodes.

The ellipsometer is used to measure the circular polarisation $S_{3}$ of the laser beam of any unknown polarisation by varying the azimuthal angle of the plate and analysing the intensities emerging from the prism.

\subsubsection{Ellipsometer components}

The HBS: The beam sampler is a hologram in engraved relief on a transparent substrate (silica) inducing forward diffraction. The birefringence of the HBS alone has been measured before its installation in the cavity system and the result is compatible with zero [12].

The Wollaston prism: The most important characteristic of the prism is its extinction rate, which is less than a few $10^{-5}$ as given by the manufacturer and has been confirmed experimentally.

The quarter wave plates: As will be described in Sect. 2.3, two QWPs $\mathrm{pl}_{1}$ and $\mathrm{pl}_{2}$ with different nominal thicknesses of $e_{\text {nom }}^{(1)}=91.2 \mu \mathrm{m}$ and $e_{\text {nom }}^{(2)}=639.9 \mu \mathrm{m}$ are used in the ellipsometer for its characterisation in order to increase the constraints of the system. Each QWP is a parallel plate of high quality quartz manufactured especially for the ellipsometer characterisation purpose, and has a delay tolerance of $1 / 300$ and a thickness tolerance of a few micrometers with a parallelism between the two faces of the order of 10 seconds of arc. The optical axis is contained in the plane of the plate.

The detection system: The detection system consists of three photodiodes $\mathrm{pd}_{0}, \mathrm{pd}_{1}$ and $\mathrm{pd}_{2}$ made of a gallium arsenide and indium alloy (InGaAs). Each photodiode and their electronics are thermalised with a Peltier module to prevent temperature variations which might be of a few degrees in the HERA environment and could induce a variation in the photodiode readout larger than the required precision. Using Peltier modules, a stability level of tenth of a degree is achieved. The photodiodes are read out with 12-bit analog-to-digital converters at a maximum sample rate of $2 \mathrm{MHz}$. For each measurement, the mean value over ten thousand signals $I_{\mathrm{pd}}$ is recorded after subtraction of a pedestal Ped for each photodiode. The effect of laser power variations is taken into account by normalising the measurement to that of the reference photodiode $\mathrm{pd}_{0}$. Explicitly, 
photodiode intensities $I_{1}$ and $I_{2}$ used in the following can be written as:

$$
I_{1,2}=\frac{\left\langle I_{\mathrm{pd}_{1,2}}\right\rangle_{10000}-\operatorname{Ped}_{1,2}}{\left\langle I_{\mathrm{pd}_{0}}\right\rangle_{10000}-\operatorname{Ped}_{0}}
$$

A study of photodiodes in an optical laboratory has shown that in such a clean environment, the temperature regulation, the subtraction of photodiode pedestals and the laser power variation effect can be controlled such that $I_{1}$ and $I_{2}$ are known at the per mill level. The HERA accelerator environment is more noisy; effects such as larger temperature variations and the presence of quadrupoles and dipoles, the synchrotron radiation, accelerating cavities and long cables affect the precision of the photodiode measurements. This is illustrated in Figs. 2(a) and (b) where the distributions of $I_{2}$ from two data samples recorded in the HERA tunnel at different moments are shown. Each entry in the histograms is a measurement of $I_{2}$ as defined in Eq.(2.1) and the duration of data taking for each sample was approximately fifteen minutes. Fig. D(a) represents a well clustered distribution whereas Fig. 2(b) shows two populations. To take into account this kind of drift, the corresponding
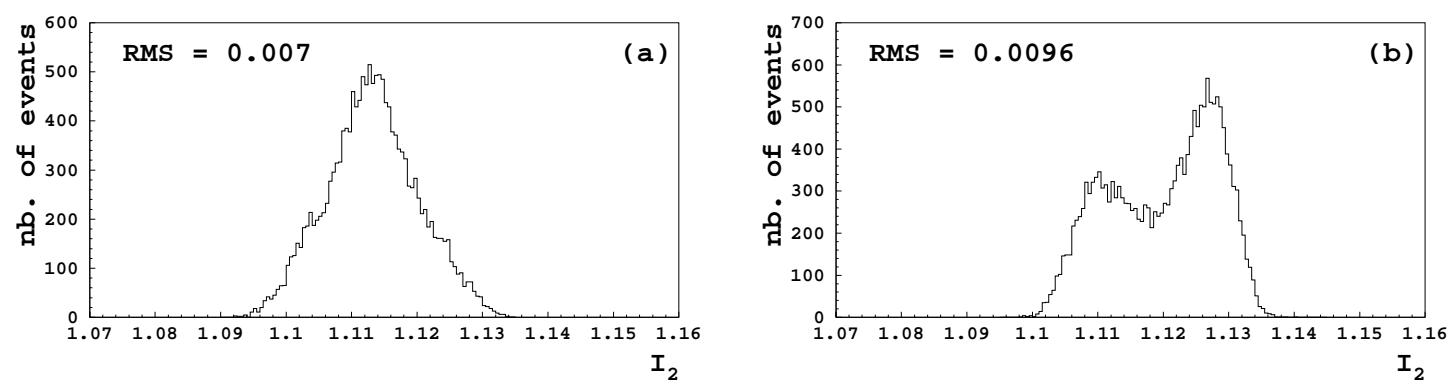

Figure 2. Histograms of $I_{2}$ as defined in Eq.(2.1) for two data sets (a) and (b) taken during fifteen minutes each at different moments.

error $\sigma_{I_{1,2}}$ is defined as the root mean square (RMS) value of the histogram, whether it has a Gaussian shape or not. In this way, the unknown long term effects such as those illustrated in Fig. 2(b) are included in the measurement errors. The errors are then parameterised as a function of the intensities as:

$$
\sigma_{I_{1,2}=A_{1,2}} I_{1,2}+B_{1,2}
$$

Fig. 3 shows the errors $\sigma_{I_{1}}$ (a) and $\sigma_{I_{2}}$ (b) as a function of $I_{1}$ and $I_{2}$ respectively and the corresponding parameterisation of Eq.(2.2), for data sets recorded at various azimuthal angles of the ellipsometer QWP in order to cover the entire range of intensity values. These errors may depend on the duration of acquisition time which will be varied for systematics studies (Sect. 3.1).

The accurate measurement of the degree of circular polarisation $S_{3}$ of the laser beam requires a precise control and characterisation of this ellipsometer. Therefore before determining $S_{3}$, the ellipsometer will be first used as a calibration system to characterise precisely some of its optical components. For this, a complete simulation model has been developed. 

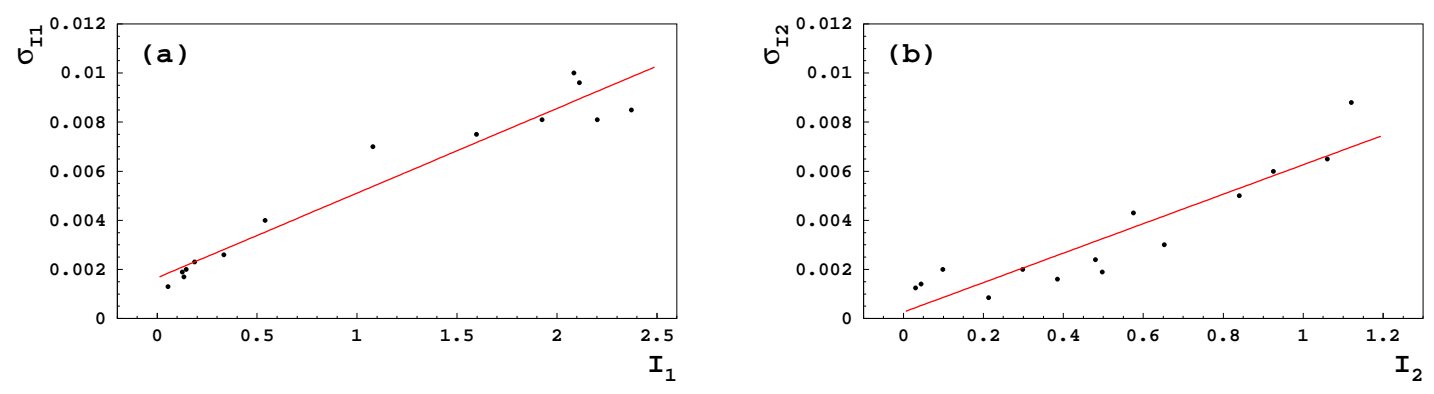

Figure 3. Errors $\sigma_{I_{1}}$ (a) and $\sigma_{I_{2}}$ (b) as a function of $I_{1}$ and $I_{2}$, respectively. The lines correspond to straight line fits to the measured errors according to Eq.(2.2).

\subsection{Model of the ellipsometer and $\chi^{2}$ function}

\subsubsection{Model of the ellipsometer}

The polarisation state $\mathbf{E}_{\text {elli }}$ at the entrance of the ellipsometer and the associated degree of circular polarisation $S_{3}^{\text {elli }}$ can be written in the most general form as functions of two angles $\xi_{\text {elli }}$ and $\phi_{\text {elli }}$ as [13]:

$$
\mathbf{E}_{\text {elli }}=\left(\begin{array}{l}
\cos \xi_{\text {elli }} \\
\sin \xi_{\text {elli }} e^{i \phi_{\text {elli }}}
\end{array}\right), \quad S_{3}^{\text {elli }}=2 \cos \xi_{\text {elli }} \sin \xi_{\text {elli }} \sin \phi_{\text {elli }}
$$

The expression of the two transmitted fields $\mathbf{E}_{1}$ and $\mathbf{E}_{2}$ emerging from the Wollaston prism and the associated theoretical intensities $T_{1}$ and $T_{2}$ can then be written as:

$$
\mathbf{E}_{1(2)}=\mathscr{M}_{1(2)}^{\text {elli }} \mathbf{E}_{\text {elli }}, \quad T_{1(2)}=\left|\mathbf{E}_{1(2)}\right|^{2},
$$

where $\mathscr{M}_{1}^{\text {elli }}$ and $\mathscr{M}_{2}^{\text {elli }}$, standing for the corresponding Jones transmission matrices [14], are computed from a theoretical model as follows:

The Wollaston cube: Two small parameters $\varepsilon_{x}$ and $\varepsilon_{y}$ are introduced to take into account in the Wollaston Jones matrix a possible small birefringence along its two optical axes [15].

The quarter wave plate: The model used for the QWP takes into account the multiple reflections of the wave inside a quartz plate of indices $n_{o}$ and $n_{e}$, of thickness $e_{\mathrm{QWP}}$, and whose optical axis has an angle $\phi_{\mathrm{oa}}$ with respect to the laboratory frame. This angle has two components: $\phi_{\mathrm{oa}}=\phi+\phi_{0}$, where $\phi$ is the azimuthal angle of the QWP motorised rotating stage and $\phi_{0}$ is an azimuthal reference angle reflecting the fact that the orientation of the optical axis in the plane of the plate is not a priori at position $\phi=0$. The simulation also models the passage of a light wave through the plate at a non zero incident angle $\theta_{\text {inc }}$, which is equal to the number of tilting stage screw turns $\left(X_{\text {turn }}\right)$ times the tilt angle value of one screw turn $\left(\theta_{\text {turn }}\right)$. The light beam is treated as a plane wave since, at small incident angles (i.e. less than $0.1 \mathrm{rad}$ for the work described here), the comparison between a plane wave treatment and a Gaussian wave treatment shows that the Gaussian character of the wave can be neglected [16]. Also, the contribution of the optical activity of the crystal [17] as well as the surface roughness [18] has been studied and found to be negligible (a relative contribution of less than $10^{-6}$ ). 
Misalignments: A realistic description of the optical system must also take into account the following experimental misalignments:

- A misalignment of the QWP plane with respect to the Wollaston prism axes, which is modeled by a small tilt angle $\delta_{W}$ of the normal to the QWP.

- A misalignment due to the fact that the laser beam may not enter the QWP exactly at the plate center of the mount: in that case, because the two plate surfaces may not be perfectly parallel, the thickness crossed by the light can vary during an azimuthal rotation of the plate. To take into account this effect, the plate is modeled by a variable thickness as: $e=e_{\mathrm{QWP}}[1+$ $\left.\left(\delta_{c} / e_{\mathrm{QWP}}\right) \cos \left(\phi-\phi_{c}\right)\right]$ where $e_{\mathrm{QWP}}$ is the thickness of the plate in case of a perfect alignment situation, $\delta_{c}$ represents a small shift between the laser beam impact point and the plate center of the mount, and $\phi_{c}$ is an arbitrary reference azimuthal angle, as the position $\phi=0$ of the QWP may not be the position which maximises the plate thickness $e$. One new parameter $\delta_{c}$ is introduced each time the incident angle $\theta_{\text {inc }}$ changes.

According to this model of ellipsometer components and misalignments, for a given ellipsometer QWP plate $\mathrm{pl}_{k}$ of thickness $e_{\mathrm{QWP}}^{k}$, a given incident angle $\theta_{\mathrm{inc}}^{j}$, azimuthal angle $\phi^{i}$ of the plate, and polarisation state $\mathbf{E}_{\text {elli }}^{\ell}$, the two theoretical intensities $\mathrm{T}_{1(2)}$ of Eq.(2.4) can be written as functions of ellipsometer parameters as:

$$
\begin{array}{r}
T_{1(2)}^{i j k \ell} \equiv f_{1(2)}\left(n_{o}, n_{e}, \varepsilon_{x}, \varepsilon_{y}, e_{\mathrm{QWP}}^{k}, \theta_{\mathrm{turn}} \times X_{\mathrm{turn}}^{j},\right. \\
\left.\phi_{0}^{k}+\phi^{i}, \xi_{\mathrm{elli}}^{\ell}, \phi_{\mathrm{elli}}^{\ell}, \delta_{W}^{k}, \delta_{c}^{j}, \phi_{c}^{k}\right) .
\end{array}
$$

All the details of the model described here can be found in [19] where the modeling and the calculations relative to the Wollaston cube, the QWP and the optical misalignments are explicitly given. In particular, the calculation of the Jones transmission matrix of a quartz plate at a non zero incident angle, with internal reflections being taken into account, is explicitly performed in the appendix of [19]. Simulations show that multiple reflections, plate defects or optical misalignments contribute at the few percent level to the transmitted intensities $T_{1(2)}$. Achieving the per mill level for $S_{3}$ measurement is therefore only possible using this complete model description.

\subsection{2 $\chi^{2}$ function}

To characterise the ellipsometer and distinguish effects due to optical misalignments from those due to plate defects or those due to the light polarisation state, the principle is to record ellipsometer experimental data $I_{1(2)}$ (as defined in Eq. 2.1) ) and to minimise the following $\chi^{2}$ function:

$$
\begin{aligned}
\chi^{2}= & \sum_{k=1, N_{\mathrm{pl}}} \sum_{\ell=1, P_{k}} \sum_{j=1, \Theta_{k}} \sum_{i=1, N_{\phi}} \\
& {\left[\left(\frac{R_{1}^{j \ell k} T_{1}^{i j \ell k}-I_{1}^{i j \ell k}}{\sigma_{1}^{i j \ell k}}\right)^{2}+\left(\frac{R_{2}^{j \ell k} T_{2}^{i j \ell k}-I_{2}^{i j \ell k}}{\sigma_{2}^{i j \ell k}}\right)^{2}\right], }
\end{aligned}
$$

where $N_{\mathrm{pl}}$ refers to the number of the QWP used in the data taking, $P_{k}$ to the number of polarisation states, $\Theta_{k}$ to the number of incident angles and $N_{\phi}$ to the number of azimuthal angles of the QWP.

$T_{1(2)}^{i j \ell k}\left(I_{1(2)}^{i j \ell k}\right)$ is the theoretical (experimental) photodiode intensity calculated (measured) when the 
plate $\mathrm{pl}_{k}$ is inserted in the ellipsometer, at the $\ell^{\text {th }}$ polarisation state $\mathbf{E}_{\text {elli }}$, the $j^{\text {th }}$ value of $\theta_{\text {inc }}$ and the $i^{\text {th }}$ value of $\phi . \quad \sigma_{1(2)}^{i j \ell k}$ is the uncertainty of $I_{1(2)}^{i j \ell k}$ in Eq.(2.2). $R_{1(2)}^{j \ell k}$ are normalisation factors which are easily determined by solving $\partial \chi^{2} / \partial R=0$. The minimisation of the $\chi^{2}$ (Eq.(2.6)) leads to parameter values of Eq.(2.5), excepted for $X_{\text {turn }}^{j}, \phi^{i}$ and $n_{e} . X_{\text {turn }}^{j}$ and $\phi^{i}$ take some known values of the tilting and rotating stages, respectively. The extraordinary index $n_{e}$ is derived from previous studies [20, 21], where the quartz indices were measured at a few $10^{-5}$ level, because our constraints are not sufficient to determine $n_{o}$ and $n_{e}$ at the same time, and so the quantity that is determined in practice is the birefringence $n_{e}-n_{o}$. The determination of $n_{o}$ by the ellipsometer does not aim for the same level of precision as was obtained in [20, 21] but provides a good test of the validity of the model.

\subsection{Ellipsometer parameter determination}

The determination of ellipsometer parameters (Eq. (2.5) has been performed twice, independently in two different environments corresponding to the optical laboratory and the HERA tunnel, with two different data taking procedures.

The first data sample recorded in the optical laboratory is devoted to determine all ellipsometer characteristics and in particular the thickness of the QWP and the index $n_{o}$. The determination of both $e_{\mathrm{QWP}}$ and $n_{o}$ requires a long and meticulous data taking procedure since it turns out that the solution of the $\chi^{2}$ minimisation is not unique: several combinations $\left(e_{\mathrm{QWP}}, n_{o}\right)$ can minimise the $\chi^{2}$. In order to resolve such an ambiguity, two uncoated plates $\mathrm{pl}_{1}$ and $\mathrm{pl}_{2}$ with different thicknesses are used by inserting one after the other in the ellipsometer. In addition, for each plate, several data sets are taken for different incident angles.

The second data sample was recorded after the installation of the setup in the HERA tunnel in order to characterise again the system, since all the optical components were dismounted to be transported from the optical laboratory to the tunnel. In the tunnel, data taking conditions were much more difficult than in the optical laboratory, and in particular, the tunnel accesses were limited to a few hours per month. The corresponding data taking procedure thus has to be simpler. To avoid ambiguous solutions on the thickness of plate $\mathrm{pl}_{1}$, its fit range is restricted to around the expected value obtained from the laboratory calibration.

\subsubsection{The data calibration samples}

The first calibration of the ellipsometer was performed in the clean optical laboratory, where the room temperature was regulated at $25^{\circ}$. For a given laser beam polarisation state, ellipsometer measurements were recorded for each plate $\mathrm{pl}_{1}$ and $\mathrm{pl}_{2}$ at various incident angles $\theta_{\text {inc }}$ between the laser beam and the plate normal direction, and, for each $\theta_{\text {inc }}$, the QWP was turned azimuthally of an angle $\phi$ in step of $1^{\circ}$ from $0^{\circ}$ to $360^{\circ}$. A Monte Carlo study shows that to provide enough constraints to minimise the $\chi^{2}$ and determine all the ellipsometer parameters of Eq.(2.5), data have to be recorded at four (two) different values of $\theta_{\text {inc }}$ for the QWP pl ${ }_{1}\left(\mathrm{pl}_{2}\right)$, and it is sufficient to have only one polarisation state of a given $\left(\xi_{\text {elli }}, \phi_{\text {elli }}\right)$. Each time the incident angle $\theta_{\text {inc }}$ was changed, a long procedure was applied to displace manually the plate transversally with the linear stage micrometric screws in order to recover precisely the matching of the plate mechanical center with the laser impact point. The six samples recorded at different values of $\theta_{\text {inc }}$ containing 360 entries 
each are called $D_{d}^{\mathrm{lab}}(d=1, \cdots, 6)$ and, when introduced in the $\chi^{2}$ function, values of subscripts in Eq.(2.6) are $N_{\mathrm{pl}}=2, P_{1}=1, P_{2}=1, \Theta_{1}=4, \Theta_{2}=2$ and $N_{\phi}=360$.

The second data set was recorded in the HERA tunnel where the room temperature was regulated at $35^{\circ}$. In order to control the data taking procedure from outside tunnel to be independent of the short duration of tunnel access, only one plate $\left(\mathrm{pl}_{1}\right)$ was used in the ellipsometer and the incident angle $\theta_{\text {inc }}$ between the light beam and this QWP remained fixed and equal to zero. In this configuration, the $\chi^{2}$ minimisation was performed by using three recorded data samples, each with a different azimuthal angle of the entrance plate $\mathrm{QWP}_{\text {ent }}$. In this way, the light entering the ellipsometer has three different polarisation states. For each of these three $\mathrm{QWP}_{\mathrm{ent}}$ azimuthal positions, the ellipsometer QWP was also turned azimuthally through an angle $\phi$ in step of $1^{\circ}$ from $0^{\circ}$ to $360^{\circ}$. These three data samples are called $D_{d}^{\mathrm{HERA}}(d=1, \cdots, 3)$ and the corresponding values for the superscripts in Eq.(2.6) are: $N_{\mathrm{pl}}=1, P_{1}=3, \Theta_{1}=1$ and $N_{\phi}=360$.

\subsubsection{Correlation between $e_{\mathrm{QWP}}$ and $S_{3}$}

Among all ellipsometer parameters, the dominant source of systematic error on $S_{3}$ comes from the QWP thickness uncertainty. Thus, before giving the results of the minimisations using the two sets of data samples previously described, it is interesting to show the correlation between the plate thickness $e_{\mathrm{QWP}}$ and $S_{3}$. The effect on the $S_{3}$ determination is estimated by simulating an ellipsometer data sample with a degree of circular polarisation $S_{3}^{\text {true }}$ and a plate thickness $e_{\text {gen }}$. Using this sample, various minimisations of the $\chi^{2}$ are performed by letting only $S_{3}^{\text {elli }}$ free (i.e. only the two angles $\xi_{\text {elli }}$ and $\left.\phi_{\text {elli }}\right)$, by fixing the plate thickness to different values $e_{\text {fix }}$ slightly different from $e_{\text {gen }}$, and by fixing all the other parameters to their generated values. The quantity $\left|\left(S_{3}^{\text {true }}-S_{3}^{\text {elli }}\right) / S_{3}^{\text {true }}\right|$ presented in Fig. $\bigoplus$ as a function of $e_{\text {fix }}-e_{\text {gen }}$ shows that a systematic error of one micometer on the plate thickness leads to a systematic error around $0.5 \%$ on $S_{3}$. A precise knowledge of the plate thickness inside the thickness tolerance of a few micrometers given by the manufacturer has therefore to be reached to keep a systematic error at the per mill level on the measurement of $S_{3}$. Achieving this precision is only possible using the complete model described previously.

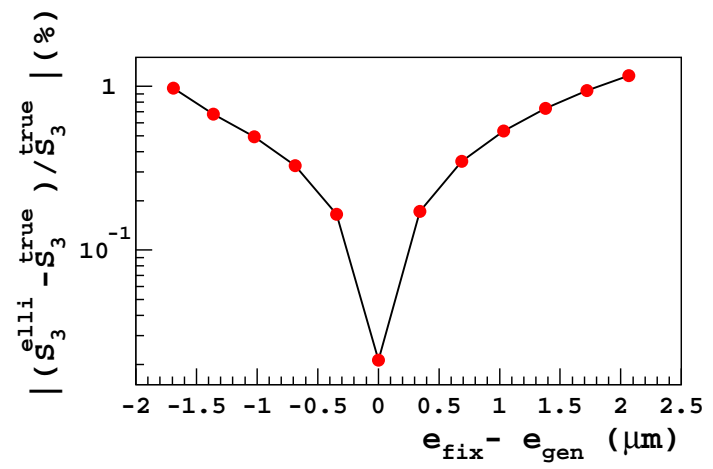

Figure 4. Relative error on $S_{3}^{\text {elli }}$ as a function of the uncertainty on the ellipsometer QWP thickness. 


\subsubsection{Results}

The $\chi^{2}$ minimisation procedure was tested first by using simulated samples generated with Eq.(2.4) following closely the experimental data. It was found that all fitted parameters were in agreement with the generated ones within a precision of a few per mill. The minimisation is then performed independently with the two experimental data sets $D_{d}^{\mathrm{lab}}$ and $D_{d}^{\mathrm{HERA}}$ and leads to values of $\chi^{2}$ per degree of freedom equal to 1.07 and 2.09 respectively. The excellent agreement between experimental intensities and theoretical ones based on the fit is illustrated by a typical example in Fig. 5, where the quantities $I_{1,2}$ and $R_{1,2} T_{1,2}$ are presented for the sample $D_{1}^{\mathrm{HERA}}$ as a function of the azimuthal angle $\phi$ of the ellipsometer QWP.
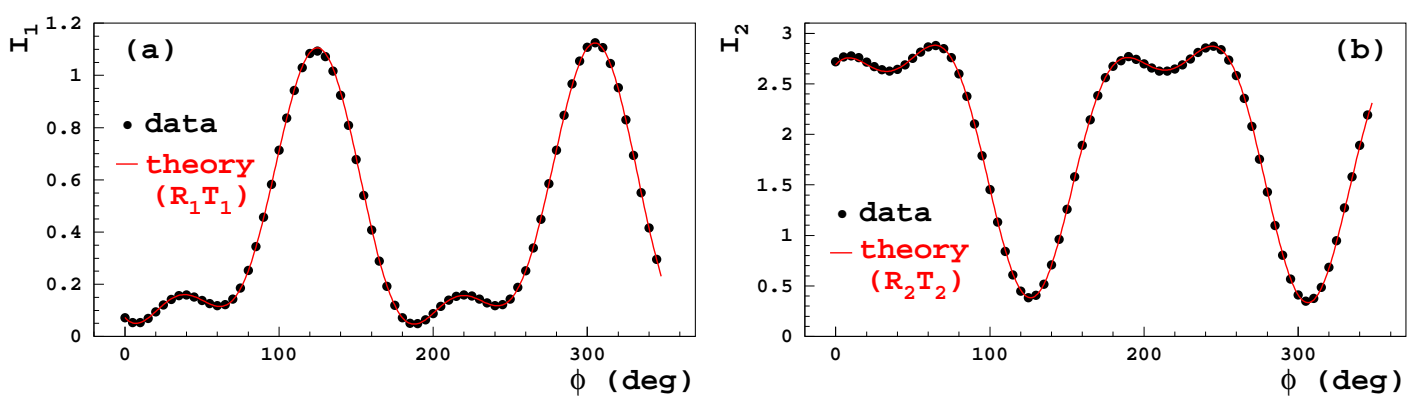

Figure 5. Experimental intensities (black bullets, for clarity only a subsample is shown) $I_{1}$ (a) and $I_{2}$ (b) compared with theoretical ones (curves) derived from the fit, as a function of the azimuthal angle $\phi$ of the ellipsometer QWP, for the data file $D_{1}^{\mathrm{HERA}}$.

All ellipsometer parameters determined from the two minimisations using the data sets $D_{d}^{\text {lab }}$ or $D_{d}^{\mathrm{HERA}}$ are found to be realistic and well defined. Among them, one interesting quantity is the quartz birefringence value $n_{e}-n_{o}$ which can be compared with textbook values previously determined. In [21, 22], birefringence measurements were performed at a temperature of $18^{\circ}$ and $22^{\circ}$. Our measurements in the optical laboratory and in the tunnel were carried out at higher temperatures of $25^{\circ}$ and $35^{\circ}$, respectively. Based on the relation of optical index variation with temperature [23], quartz birefringence values of [21, 22] are scaled to $T=25^{\circ}$ and at $T=35^{\circ}$ and are shown in Fig. 6 together with the two birefringence values $n_{e}-n_{o}$, where the $n_{o}$ is obtained from the fits and the $n_{e}$ (and its uncertainty of $\sim 2 \times 10^{-5}$ ) taken from Refs. [20, 21]. Our results agree at better than one per mill with the ones quoted in the references.

As for the birefringence, all other results determined in the optical laboratory are compared with those in the tunnel and good agreement are found once the effects of temperature difference and optical alignment difference are taken into account [19].

\section{Regular measurements of $S_{3}$ and systematics studies}

\subsection{Measurements and systematic uncertainty from the ellipsometer}

During the data taking period of the cavity polarimeter, when the cavity was locked in resonant 


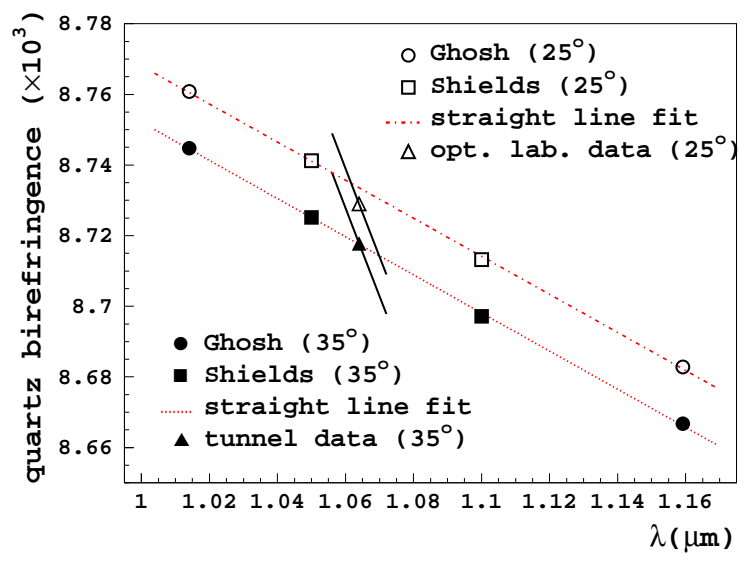

Figure 6. Quartz birefringence values scaled to $25^{\circ}$ and $35^{\circ}$ from textbook values [21, 22], as a function of the wavelength. Dashed lines are straight line fits to four scaled textbook points. Birefringence values determined from the fits to data $D^{\mathrm{lab}}$ and $D^{\mathrm{HERA}}$ are indicated by an open and a solid triangle, respectively. The error bars are inclined for clarity.

state and the azimuthal angle $\phi_{\text {ent }}$ of the entrance QWP was such that the light is close to a fully right or left circularly polarised state $S_{3}= \pm 1$ [1], values of $S_{3}^{\text {elli }}$ were regularly determined.

Each value of $S_{3}^{\text {elli }}$ is extracted from a data sample recorded with the ellipsometer and containing 180 photodiode signals (as defined in Eq.(2.1)) corresponding to a $2^{\circ}$-step azimuthal turn of the ellipsometer QWP. The duration of data taking was about ten minutes for each sample.

To extract $S_{3}^{\text {elli }}$ and its uncertainty $\sigma_{S_{3} \text { elli }}$, the $\chi^{2}$ function defined in Eq. (2.6) is minimised. The only fitted parameters are the polarisation state parameters $\xi_{\text {elli }}$ and $\phi_{\text {elli }}$ and all other parameters are fixed to values previously determined by the characterisation of the tunnel optical system as described in Sect. 2 . Thanks to a heat regulation system, the temperature inside the isotherm house (see [1]) was controlled within $\pm 0.3^{\circ} \mathrm{C}$ which ensured a perfect stability of the optical axis (defined by the positions of the cavity mirrors) over time and therefore the stability of our ellipsometer calibration.

The uncertainty $\sigma_{S_{3}^{\text {elli }}}$ obtained from the $\chi^{2}$ minimisation is of the order of a few $10^{-4}$. During one year (from June 2006 to June 2007), the azimuthal positions of the plate QWP $_{\text {ent }}$ defining a left or right circularly polarised laser beam were changed only three times, either after an hardware problem on the rotating mount controller of the plate, or after a dedicated check involving the plate or the photodiode $\mathrm{pd}_{\text {ent }}$. The $S_{3}^{\text {elli }}$ measurements were very stable over time since, over the one-year period, they have shown a stability of a few per mill [19].

Given the precise ellipsometer calibration procedure described in Sect. 2.2, the only remaining source of systematic uncertainty on $S_{3}^{\text {elli }}$ concerns the duration of the data taking. Indeed, the duration of one measurement sample taken with the ellipsometer can have an effect on the photodiode intensity distributions because of long term fluctuation as shown in Fig. 2(b). This duration depends on the chosen interval between two consecutive azimuthal angles $\phi$ of the ellipsometer QWP. To study this effect, the entrance plate $\mathrm{QWP}_{\text {ent }}$ was fixed to a given azimuthal position and 
several measurement samples were recorded using the ellipsometer for different durations ranging from three to twenty minutes. The extracted $S_{3}^{\text {elli }}$ values from these samples are found to be compatible within two per mill [19]. To be conservative, an error of three per mill is quoted for the uncertainty due to the duration of data taking.

\subsection{Transport of $S_{3}$ from ellipsometer to cavity center}

The precise $S_{3}$ values obtained above concern the degree of circular polarisation at the entrance of the ellipsometer. What we are interested in is, however, the $S_{3}$ value at the electron-laser interaction point, i.e. at the center of the Fabry-Perot cavity. A priori, these two values are the same, but because of the presence of optical components between the two, a small difference could be induced. The transport of $S_{3}$ and its uncertainty are the subject of this section.

\subsubsection{Parasitic ellipticity from cavity center to ellipsometer entrance}

Between the center of the cavity and the entrance of the ellipsometer (see Fig. 11), the exit mirror and the exit window of the Fabry-Perot cavity and the optical system $M_{T}$ could be a source of birefringence and may induce parasitic ellipticity, modifying the laser beam polarisation. The birefringence of the substrate, the coating and the mounting system of the exit mirror and the exit window has been estimated or measured from dedicated studies [24, 19]. It was shown that the bias induced on $S_{3}$ from the center to the exit of the cavity is at the utmost of the order of $3 \times 10^{-5}$. The remaining dominant source of parasitic ellipticity is associated to the $45^{\circ}$ dielectric mirrors used to guide the light into the ellipsometer because of their different reflection coefficients for two electric field components: one in the incident plane and the other perpendicular to it [13, 25]. To determine this ellipticity, the transfer matrix $M_{T}$ of the system has to be determined.

\subsubsection{Exit transfer matrix $M_{T}$}

An optical theorem demonstrated by R. Clark Jones [9] states that any optical system composed of any non-absorbing components may always be replaced by a partial polariser placed between two delay plates, with the addition of a rotator inserted at any position in the system. Under the assumption that no power is absorbed in optical materials, the Jones matrices of a partial polariser, a delay plate and a rotator $P_{p_{1} p_{2}}, G_{\gamma}$ and $R_{\theta}$ can be written as [6]:

$$
P_{p_{1} p_{2}}=\left(\begin{array}{cc}
p_{1} & 0 \\
0 & p_{2}
\end{array}\right), \quad G_{\gamma}=\left(\begin{array}{cc}
e^{i \gamma} & 0 \\
0 & e^{-i \gamma}
\end{array}\right), \quad R_{\theta}=\left(\begin{array}{cr}
\cos \theta & -\sin \theta \\
\sin \theta & \cos \theta
\end{array}\right) .
$$

Following the Jones theorem [9] and starting with a completely linear polarisation state $\mathbf{E}_{G}=$ $\left(\cos \phi_{G}, \sin \phi_{G}\right)^{T}$, the theoretical final state $\mathbf{E}_{\text {th }}$ after passing through an optical system of transfer matrix $M_{T}$ can then be modeled as:

$$
\begin{array}{ll} 
& \mathbf{E}_{\text {th }} \equiv\left(\cos \xi^{\text {th }}, \sin \xi^{\text {th }} e^{i \phi^{\text {th }}}\right)^{T}=M_{T} \mathbf{E}_{G} \\
\text { with } & M_{T}=R_{\theta_{1}} G_{\gamma_{1}} R_{\theta_{2}} P_{p_{1} p_{2}} R_{\theta_{3}} G_{\gamma_{2}} .
\end{array}
$$

In order to apply this theorem to determining the matrix $M_{T}$ of the two mirrors and the HBS located at the exit of the Fabry-Perot cavity, a special configuration of the system was set up by

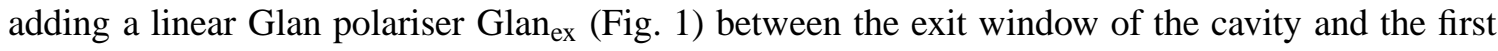


mirror. Such a device polarises the beam in a completely linear state $\mathbf{E}_{G}$ before it enters the optical elements of the exit line. In this configuration, a number of data samples $N_{d}$ were recorded using the ellipsometer, each with a different azimuthal angle $\phi_{G}^{\ell}\left(\ell=1, \cdots, N_{d}\right)$ of the polariser $\mathrm{Glan}_{\mathrm{ex}}$. For each $\phi_{G}^{\ell}$, the ellipsometer QWP was rotated azimuthally from $0^{\circ}$ to $360^{\circ}$.

A fit to these data samples is performed by minimising the $\chi^{2}$ function defined in Eq.(2.6) to obtain the only free parameters $\xi_{\text {elli }}^{\ell}$ and $\phi_{\text {elli }}^{\ell}\left(\ell=1, \cdots, N_{d}\right)$ of the beam polarisation state after the HBS. Using the resulting $\xi_{\text {elli }}^{\ell}$ and $\phi_{\text {elli }}^{\ell}$ and their uncertainties $\sigma_{\xi_{\text {elli }}^{\ell}}$ and $\sigma_{\phi_{\text {elli }}^{\ell}}$, the following $\chi^{2}$ function is constructed in order to determine the elements of the matrix $M_{T}$ :

$$
\chi^{2}=\sum_{\ell=1, N_{d}}\left[\left(\frac{\xi_{\mathrm{th}}^{\ell}-\xi_{\mathrm{elli}}^{\ell}}{\sigma_{\xi_{\mathrm{elli}}^{\ell}}}\right)^{2}+\left(\frac{\phi_{\mathrm{th}}^{\ell}-\phi_{\mathrm{elli}}^{\ell}}{\sigma_{\phi_{\mathrm{elli}}^{\ell}}}\right)^{2}\right],
$$

where $\xi_{\mathrm{th}}^{\ell}$ and $\phi_{\mathrm{th}}^{\ell}$ are the theoretical angles defining the $\ell^{\mathrm{th}}$ polarisation state after the HBS. By using Eqs.(3.1), 3.2), these angles can be written as functions of the parameters $\theta_{1}, \theta_{2}, \theta_{3}, \gamma_{1}, \gamma_{2}, p_{1}$ and $p_{2}$ of the matrix $M_{T}$ and of the angle $\phi_{G}^{\ell}$ of the linear initial polarisation state. A Monte Carlo study of the $\chi^{2}$ function defined in Eq.(3.3) shows that some elements of the matrix $M_{T}$ are completely correlated and that the system can be described only with one delay plate, one partial polariser and two rotators. The parameters of $M_{T}$ in Eq.(3.2) are therefore restricted to $\gamma_{1} \equiv \gamma_{T}, p_{1} \equiv p_{T}, p_{2}=1$, $\theta_{3}=0$ and $\gamma_{2}=0$. The minimisation of the $\chi^{2}$ defined in Eq.(3.3) leads to $\gamma_{T}=(13.1 \pm 1.4) \mathrm{mrad}$ and $p_{T}=1.001 \pm 0.001$, thereby showing that the exit optical system behaves like a delay plate inducing a birefringence of the order of a few tens of mrad.

A cross-check of this study has been performed by placing the additional polariser Glan $_{\mathrm{ex}}$ between the HBS and the ellipsometer QWP. As previously, ellipsometer data sets were recorded for several azimuthal angles of the polariser. Applying the same procedure, the matrix $M_{T}$ is now expected to be compatible with the identity matrix, since there is no optical component between the Glan and the entrance of the ellipsometer. The result of the fit, with an angle of $(1.5 \pm 4.0) \mathrm{mrad}$ for the delay plate and a value of $1.004 \pm 0.005$ for the partial polariser parameter, constitutes a valuable check of the robustness of our model describing the exit beam line of the Fabry-Perot cavity.

\subsection{3 $S_{3}$ at the exit of the cavity}

Removing the polariser Glan $_{\text {ex }}$ to recover the standard setup of the optical system, the degree of circular polarisation $S_{3}^{\text {ex }}$ at the exit of the cavity has now to be determined as a function of $S_{3}^{\text {elli }}$ at the entrance of the ellipsometer (Sect. 3.1). The polarisation state $\mathbf{E}_{\mathrm{ex}}$ at the exit of the cavity is related to $\mathbf{E}_{\text {elli }}$ (Eq.(2.3) as $\mathbf{E}_{\mathrm{ex}}=M_{T}^{-1} \mathbf{E}_{\text {elli. }}$. The development of this field expression leads to the relation:

$$
S_{3}^{e x}=S_{3}^{\text {elli }}+\delta S_{\mathrm{ex}}\left(\theta_{1}, \theta_{2}, \gamma_{T}, p_{T}, \xi_{\mathrm{elli}}, \phi_{\mathrm{elli}}\right) .
$$

The relation (3.4) applied to several values of $S_{3}^{\text {elli }}$ determined by the ellipsometer shows that the correction values $\delta S_{\mathrm{ex}}$ are all below five per mill. As the HBS is not the cause of this parasitic ellipticity (see Sect. 2.1), the bias is due to the two mirrors system. This confirms a measurement performed at Saclay in 1999 for the TJNAF polarimeter [26] in which the effect of the two mirrors on the determination of $S_{3}$ was measured to be of the order of a few per mill. 
The bias $\delta S_{\mathrm{ex}}$ is calculable for each value of $S_{3}^{\text {elli }}$ and therefore does not enter as a systematic error but is explicitly determined to correct $S_{3}^{\text {elli }}$. The uncertainty $\sigma_{S_{3}^{\text {ex }}}$ of $S_{3}^{\text {ex }}$ due to the transfer from $S_{3}^{\text {elli }}$ to $S_{3}^{\text {ex }}$ are of order of a few $10^{-4}$ and has been calculated from the $M_{T}$ elements as follows: for four combinations $\left(\gamma_{T}+\sigma_{\gamma_{T}}, p_{T}+\sigma_{p_{T}}\right),\left(\gamma_{T}-\sigma_{\gamma_{T}}, p_{T}+\sigma_{p_{T}}\right),\left(\gamma_{T}+\sigma_{\gamma_{T}}, p_{T}-\sigma_{p_{T}}\right)$ and $\left(\gamma_{T}-\sigma_{\gamma_{T}}\right.$, $\left.p_{T}-\sigma_{p_{T}}\right)$, the corresponding $S_{3}^{\text {ex }}$ are extracted and $\sigma_{S_{3}}$ is taken to be the maximum difference between these four values with the central value $S_{3}^{\text {ex }}$ being calculated with parameters $\gamma_{T}$ and $p_{T}$.

\subsection{Overall $S_{3}$ uncertainty}

Summarising all the studies and results described previously, the $S_{3}$ value inside the cavity can be written by taking into account all the uncertainties of the optical system as:

$$
S_{3}=S_{3}^{\text {elli }}+\delta S_{\text {ex }} \pm \sigma_{S_{3}^{\text {elli }}} \pm \sigma_{S_{3}^{\text {ex }}} \pm \sigma_{\text {time }} \pm \sigma_{\text {trans }},
$$

where $S_{3}^{\text {elli }}$ is the degree of circular polarisation measured using the ellipsometer, $\delta S_{\text {ex }}$ is the $S_{3}^{\text {elli }}$ dependent correction factor defined in Eq. (3.4), $\sigma_{S_{3} \text { elli }}$, of the order of a few $10^{-4}$, is the uncertainty on the measurement of $S_{3}^{\text {elli }}$ using the ellipsometer (Sect. 3.1), $\sigma_{S_{3}^{\text {ex }}}$, of the order of a few $10^{-4}$, is the uncertainty on the determination of the transfer matrix $M_{T}$ (Sect. 3.2.3), $\sigma_{\text {time }} \approx 3 \times 10^{-3}$ is the conservative uncertainty associated to the duration of of data taking of an ellipsometer data sample (Sect. 3.1), and $\sigma_{\text {trans }}<3 \times 10^{-5}$ is the uncertainty related to the passage of the light through the exit cavity mirror (Sect. 3.2.1). The last two uncertainties $\sigma_{\text {time }}$ and $\sigma_{\text {trans }}$ are common to all $S_{3}$ measurements, all others vary for each measurement of $S_{3}$.

\section{Coherence of $S_{3}$ along the whole optical system}

Although the previous studies have provided values of $S_{3}$ at the electron-laser IP with an uncertainty around three per mill, the idea is now to characterise also the entrance optical elements by a matrix $M_{E}$, determine the values of $S_{3}$ at different places of the optical system to check their coherence and make sure that no additional unknown large effect could induce a bias on $S_{3}$ at the center of the cavity.

\subsection{Determination of $M_{E}$}

The entrance beam line is described with the matrix $M_{E}$ (see Fig. 1) and is composed of a glass plate, two lenses and four alignment mirrors. Following the optical theorem of R. Clark Jones [9] already used in Sect. 3.2.2, $M_{E}$ can be expressed with the same formula (see Eq.(3.2)) as for the matrix $M_{T}$. To determine $M_{E}$, the method pursued is to model the passage of the beam from the entrance Glan polariser to the entrance cavity mirror when the cavity is unlocked, followed by the retro-reflection of the beam by the cavity mirror and its passage through the Glan in the opposite direction. A reversibility theorem [6, 10] states that for a matrix $M$ describing the light path through a given system, the matrix corresponding to the light path in the opposite direction is the transposed matrix of $M$. Following this theorem and starting with a horizontal linear polarisation state $\mathbf{E}_{\text {lin }}=$ $(1,0)^{T}$ just after the entrance Glan, the expression of the retro-reflected field $\mathbf{E}_{\text {ret }}$ and the associated intensity $T_{\text {ret }}$ emerging from the Glan in the return direction can then be written as:

with

$$
\begin{aligned}
& \mathbf{E}_{\mathrm{ret}}=G_{v} M_{\mathrm{QM}}^{T} M_{m} M_{\mathrm{QM}} \mathbf{E}_{\text {lin }}, \quad T_{\mathrm{ret}}=\left|\mathbf{E}_{\mathrm{ret}}\right|^{2} \\
& M_{\mathrm{QM}}=M_{E} R_{\mathrm{QE}} M_{\mathrm{QW}} R_{\phi_{\mathrm{ent}}},
\end{aligned}
$$


where $G_{v}$ is the matrix of the Glan polariser allowing only the vertical component of the field to pass when the beam returns, $M_{\mathrm{QM}}$ is the transfer matrix of the optical line from the plate $\mathrm{QWP}_{\mathrm{ent}}$ to the last alignment mirror, $M_{m}$ is the Jones matrix of the entrance cavity mirror, $M_{E}$ is the transfer matrix to be determined, $M_{\mathrm{QW}}$ is the Jones matrix of the plate $\mathrm{QWP}$ ent , and $R_{\mathrm{QE}}$ and $R_{\phi_{\mathrm{ent}}}$ are two $2 \times 2$ rotation matrices introduced to reflect the azimuthal orientation of $\mathrm{QWP}_{\text {ent }}$ with respect to the matrix $M_{E}$ and to the Glan polariser axes, respectively.

According to this modelisation, the elements of $M_{E}$ have been determined from data recorded with the photodiode $\mathrm{pd}_{\mathrm{ent}}$ for various positions $\phi_{\mathrm{ent}}$ of the plate $\mathrm{QWP}_{\mathrm{ent}}$, by minimising the following $\chi^{2}$ function:

$$
\chi^{2}=\sum_{i=1, N_{\mathrm{ent}}}\left(\frac{R T_{\mathrm{ret}}^{i}-I_{\mathrm{ret}}^{i}}{\sigma_{I_{\mathrm{ret}}^{i}}}\right)^{2}
$$

where $N_{\text {ent }}$ is the number of different azimuthal positions $\phi_{\mathrm{ent}}, T_{\mathrm{ret}}^{i}\left(I_{\mathrm{ret}}^{i}\right)$ is the theoretical (experimental) intensity calculated with Eq.(4.1) (measured with $\mathrm{pd}_{\mathrm{ent}}$ ) at the $i^{\text {th }}$ value of $\phi_{\mathrm{ent}}, \sigma_{I_{\mathrm{ret}}}$ is the uncertainty of $I_{\text {ret }}^{i}$, and $R$ is a normalisation factor which is determined by solving $\partial \chi^{2} / \partial R=0$. When the system was conceived, the characterisation of the entrance optical line was not planned and the photodiode $\mathrm{pd}_{\text {ent }}$ was only devoted to find the azimuthal positions of the plate $\mathrm{QWP}_{\text {ent }}$ leading to a right or left circular polarisation of the laser beam. No specific study has thus been conducted to study the response and measurement uncertainties of this photodiode, and in particular no photodiode thermal regulation and no additional reference photodiode to compensate the laser power variations have been installed. A measurement $I_{\text {ret }}^{i}$ using the photodiode $\mathrm{pd}_{\mathrm{ent}}$ thus corresponds simply to the mean value over ten thousand signals recorded with a 12-bit analog-todigital converter at a sample rate of $2 \mathrm{MHz}$. The uncertainty $\sigma_{I_{\text {ret }}^{i}}$ is defined as the RMS value of the distribution of $I_{\text {ret }}^{i}$ and is of the order of one to two percent. This level of precision is not as good as the one obtained with the ellipsometer photodiodes as described in Sect. 2.1.2, and consequently, the development of a complete theoretical model to describe each optical component would not make sense here. It is therefore sufficient to consider the Glan polariser, the plate $\mathrm{QWP}_{\text {ent }}$ (which is a quartz plate treated with an anti-reflection coating) and the cavity mirror as perfect and to write the corresponding expression of the Jones matrices used in Eq.(4.1) as:

$$
G_{v}=\left(\begin{array}{ll}
0 & 0 \\
0 & 1
\end{array}\right), \quad M_{m}=\left(\begin{array}{ll}
1 & 0 \\
0 & 0
\end{array}\right), \quad M_{\mathrm{QW}}=\left(\begin{array}{cc}
1 & 0 \\
0 & e^{-i \pi / 2}
\end{array}\right) .
$$

A Monte Carlo study shows that our data are well described by using only one delay plate and one polariser. The parameters in Eq.(3.2) are therefore restricted to $\gamma_{1} \equiv \gamma_{E}, p_{1} \equiv p_{E}, p_{2}=1$, $\theta_{3}=0$ and $\gamma_{2}=0$, and the minimisation of the $\chi^{2}$ defined in Eq.(4.2) leads to $\gamma_{E}=(-32.8 \pm$ $0.5) \mathrm{mrad}$ and $p_{E}=1.17 \pm 0.01$. The effect of the matrix $M_{E}$ is clearly visible in Fig. 7 showing the distribution of the ratio $I_{\text {ret }} /\left(R T_{\text {ret }}\right)$ either for the case where the minimisation is performed (dotted line) or for the case where the matrix $M_{E}$ is fixed to the identity (full line).

\subsection{Coherence of $S_{3}$}

Using the matrices $M_{T}$ and $M_{E}$, the $S_{3}$ values at the four different locations $S_{3}^{\text {ent }}, S_{3}^{\mathrm{in}}, S_{3}^{\mathrm{ex}}$ and $S_{3}^{\text {elli }}$ indicated in Fig. 1 can now be determined for any polarisation state of the laser beam, i.e. for any azimuthal position $\phi_{\mathrm{ent}}$ of the motorised rotating mount $\mathrm{QWP}_{\mathrm{ent}}$. These four values are 


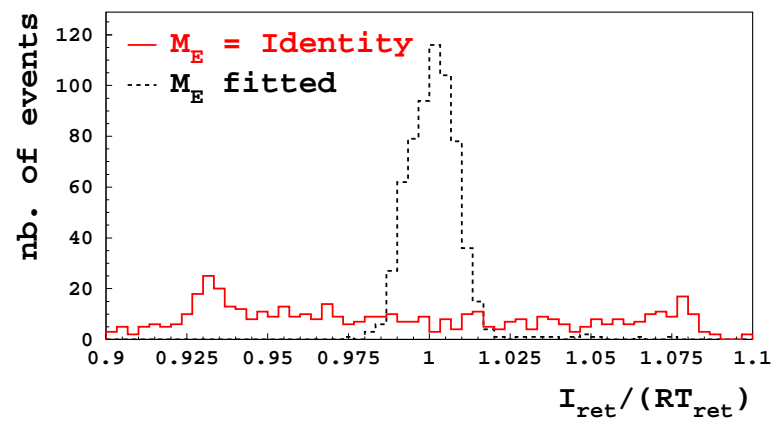

Figure 7. Ratio of the measured intensities over the theoretical ones $I_{\text {ret }} /\left(R T_{\text {ret }}\right)$ comparing the case where the $M_{E}$ is from the fit (dotted line) and the case where the $M_{E}$ is fixed to the identity (full line).

determined from their associated electric fields $\mathbf{E}_{\mathrm{ent}}, \mathbf{E}_{\mathrm{in}}, \mathbf{E}_{\mathrm{ex}}$ and $\mathbf{E}_{\mathrm{elli}}$. We recall that $\mathbf{E}_{\mathrm{ent}}$ is calculated from a linearly polarised beam passing through the plate $\mathrm{QWP}_{\text {ent }}$ which is positioned at the azimuthal angle $\phi_{\text {ent }}$ with respect to the entrance Glan axis: $\mathbf{E}_{\text {ent }}=M_{Q W} R_{\phi_{e n t}}(1,0)^{T}, \mathbf{E}_{\text {in }}$ is derived from $\mathbf{E}_{\text {ent }}$ and from the matrix $M_{E}: \mathbf{E}_{\text {in }}=M_{E} \mathbf{E}_{\text {ent }}, \mathbf{E}_{\text {elli }}$ is determined as described in Sect. 3.1 from an ellipsometer data sample, and $\mathbf{E}_{\mathrm{ex}}$ is calculated from the field $\mathbf{E}_{\text {elli }}$ and the matrix $M_{T}$ as: $\mathbf{E}_{\mathrm{ex}}=M_{T}^{-1} \mathbf{E}_{\mathrm{elli}}$.

To check experimentally the coherence of $S_{3}$ along the optical system, several arbitrarily values of $\phi_{\text {ent }}$ have been chosen close to a circularly polarised light state and, for each one of these positions, a data sample has been recorded in the ellipsometer as described in Sect. 3.1. The evolution of $S_{3}$ along the optical path can be followed in Fig. 8 through the values of $S_{3}^{\text {ent }}, S_{3}^{\text {in }}$, $S_{3}^{\text {ex }}$ and $S_{3}^{\text {elli }}$ presented for three positions $\phi_{\text {ent }}$ around a left circularly polarised state. In Fig. 8 , an uncertainty of $0.5 \%$ on the values of $S_{3}^{\text {ent }}$ is taken (typical known value as mentioned in the introduction). This uncertainty propagates directly to that of $S_{3}^{\text {in }}$.

For the measurement of the lepton beam polarisation, the only relevant quantity is the light polarisation inside the cavity, to which, of course, we do not have access but which is located between the two values $S_{3}^{\text {in }}$ and $S_{3}^{\text {ex }}$. As shown in Fig. 8 , the difference $\left|S_{3}^{\text {ex }}-S_{3}^{\text {in }}\right|$ is less than one per mill when $S_{3}^{\mathrm{ex}}$ is closer to -1 (i.e. when the system is at its operating point [1]), and can reach up to three per mill in the explored domain of $\phi_{\text {ent }}$. Part of the difference could be explained by the presence of a small birefringence due to multi-layers coating cavity mirrors as mentioned in Sect. 3.2.1. We do not know the exact value of our mirror coating birefringence, but birefringences have been measured for instance in [27, 28, 29] for cavity finesses of $6600-100000$. In all these measurements the order of magnitude of the birefringence is a few $10^{-6} \mathrm{rad}$. The Fabry-Perot cavity, with its multi-layer coating mirrors, has a finesse of about 30000 [1] and thus lies within the range quoted above. Because of the resonant optical cavity, the phase shift due to a single passage of the light in the reflected coating is amplified by a factor $2 \mathscr{F} / \pi$ [29] and becomes of the order of a few $10^{-2} \mathrm{rad}$. The bias on $S_{3}$ can be expressed in term of this amplified birefringence $\phi_{\text {bir }}$ as $S_{3}^{\text {ex }}-S_{3}^{\text {in }} \approx \phi_{\text {bir }}^{2} / 2$ [19] and can therefore be of a few per mill. However, another systematic source, which could explain the difference of a few per mill between $S_{3}^{\text {in }}$ and $S_{3}^{\text {ex }}$, is the lack of precision 


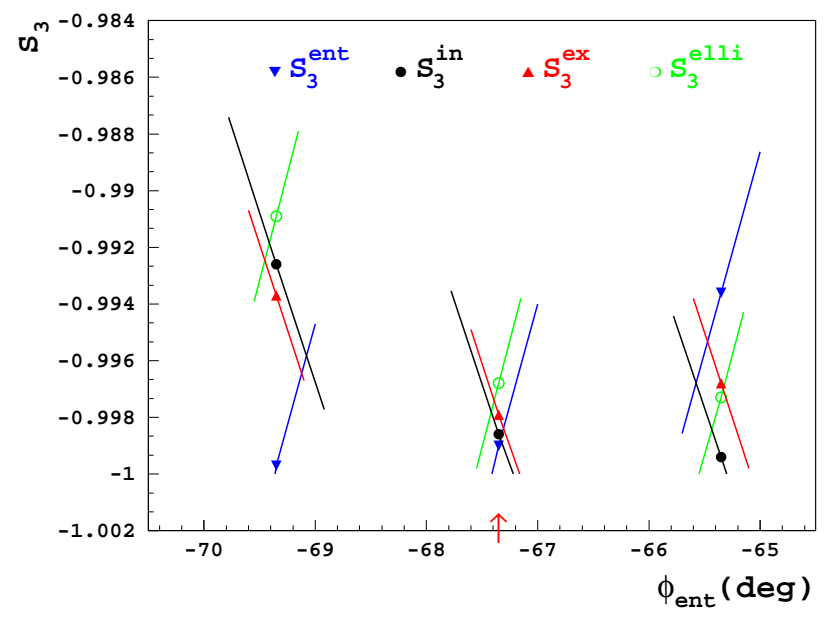

Figure 8. $S_{3}^{\text {ent }}$ (blue triangles), $S_{3}^{\text {in }}$ (black points), $S_{3}^{\text {ex }}$ (red triangles), and $S_{3}^{\text {elli }}$ (open green circles) for three azimuthal positions of the plate $\mathrm{QWP}_{\text {ent }}$ around a left circularly polarised state (indicated by an arrow). The error bars are inclined for clarity.

in measurements with $\mathrm{pd}_{\mathrm{ent}}$ used for the determination of $M_{E}$ (Sect.4.1) and thus of $S_{3}^{\text {in }}$. Anyway, the study of the entrance beam line does not intend to give an accurate measurement of $S_{3}^{\text {in }}$ but is devoted to check the coherence of the system and particularly the coherence of measurements just before and after the cavity.

\section{Summary}

The implementation of an uncoated QWP in the ellipsometer of the Fabry-Perot cavity polarimeter of HERA has allowed us to determine the degree of circular polarisation $S_{3}$ of the laser at the entrance of the ellipsometer with an uncertainty of $0.3 \%$. Such a small uncertainty is achieved thanks to a complete model description of the ellipsometer optical system. The transport of $S_{3}$ up to the electron-laser IP has then been studied and the modeling of the optical elements located between the IP and the ellipsometer has made it possible to conserve the uncertainty of $0.3 \%$ at the IP. A study of the optical line from the IP up to the laser head has also been performed and has shown that even with an unoptimised photometric measurement, $S_{3}$ is controlled along the optical path at the few per mill level. The level of accuracy presented here has, to our knowledge, never been reached in the environment of a particle collider and provides a good prospect for applications in a future linear collider [30, 31, 32].

\section{Acknowledgment}

We would like to thank T. Cacérès, M. Delbard, N. Falletto, M. Linz, A. Reboux and M. Woods for their help to this work. 


\section{References}

[1] S. Baudrand et al., A High Precision Fabry-Perot Cavity Polarimeter at HERA, JINST 5 P06005 (2010) [arXiv:1005.2741 [physics.ins-det]].

[2] R.C. King, A precise measurement of the left right asymmetry of $Z$ boson production at the SLAC linear collider, Thesis, SLAC-REPORT-452 (1994)

[3] A. Lath, A precise measurement of the left-right cross section asymmetry in $Z$ boson production, Thesis, SLAC-REPORT-454 (1994)

[4] N. Falletto, Etude, conception et réalisation d'une cavité Fabry-Perot pour le polarimètre Compton de TJNAF, Thesis Univ. J. Fourier-Grenoble 1, DAPNIA/SPhN-99-03T.

[5] HAPPEX collaboration, N. Falletto et al., Compton scattering off polarized electrons with a high-finesse Fabry-Perot Cavity at JLab, Nucl. Instr. and Meth. A 459 (2001) 412-425.

[6] R. Clark Jones, A new Calculus for the Treatment of Optical Systems. I. Description and Discussion of the Calculus, J. Opt. Soc. Am. 31 (1941) 488.

[7] J. Poirson, T. Lanternier, J.C. Cotteverte, A. Le Floch and F. Bretenaker, Jones matrices of a quarter-wave plate for Gaussian beams, App. Opt. 34 (1995) 6806-6818.

[8] M. Woods, private communication.

[9] R. Clark Jones, A new Calculus for the Treatment of Optical Systems. II. Proof of the Three General Equivalence Theorems, J. Opt. Soc. Am. 31 (1941) 493.

[10] N. Vansteenkiste, P. Vignolo, and A. Aspect, Optical reversibility theorems for polarization: application to remote control of polarization, J. Opt. Soc. Am. A 10 (1993) 2240-2245.

[11] E.D. Black, An introduction to Pound-Drever-Hall laser frequency stabilization, Am. J. Phys. 69, (2001) 79-87.

[12] M. Linz, private communication.

[13] Serge Huard, Polarisation de la lumière, Masson, Paris France (1993).

[14] R. Clark Jones, A New Calculus for the Treatment of Optical Systems. V, A More General Formulation, and Description of Another Calculus, J. Opt. Soc. Am. 37 (1947) 107-110.

[15] R.M.A. Azzam and N.M. Bashara, Ellipsometry and polarized light, Amsterdam The Netherlands (1997).

[16] F. Zomer, Transmission and reflection of Gaussian beams by anisotropic parallel plates, J. Opt. Soc. Am. A 20 (2003) 172-182.

[17] F. Zomer, Transmission matrix of a uniaxial optically active crystal platelet, Opt. Com. 252 (2005) 355-368.

[18] F. Zomer, Surface roughness effects on the transmission of Gaussian beams by anisotropic parallel plates, J. Phys. D: Appl. Phys. 36 (2003) 2697-2704.

[19] M. Jacquet, Per mill level control of the Fabry-Perot cavity optical system for precision Compton polarimetry, Habilitation thesis, LAL 09-210 (2009) [http://publication.lal.in2p3.fr/2009/hdr-MJacquet.pdf].

[20] A. Carvallo, Recherche de précision sur la dispersion infra-rouge du quartz, Comptes rendus des scéances de l'académie des sciences 126 (1989) 728. 
[21] G. Ghosh, Dispersion-equation coefficients for the refractive index and birefringence of calcite and quartz crystals, Opt. Com. 163 (1999) 95-102.

[22] J.H. Shields and J.W. Ellis, Dispersion of birefringence of Quartz in the near infrared, J. Opt. Soc. Am. 46 (1956) 263.

[23] T. Toyoda and M. Yabe, The temperature dependance of the refractive indices of fused silica and crystal quartz, J. Phys. D: App. Phys. 16 (1983) 97-100.

[24] F. Zomer, A high power Fabry-Perot resonator for precision Compton Polarimetry with the longitudinally polarised lepton beams at HERA, Habilitation thesis, LAL 03-12 (2003), [http://publication.lal.in2p3.fr/2003/LAL03.12.pdf].

[25] Warren J. Smith, Modern Optical Engineering, McGraw-Hill (2000).

[26] A. Delbart, Polarisation du faisceau de photons au CIP: Modélisation des manips en labo, application à la cavité du polarimètre, DSM/DAPNIA 6-6112 R 2000020 (1999).

[27] D. Jacob, M. Vallet, F. Bretenaker, A. Le Floch and M. Oger, Supermirror phase anisotropy measurement, Opt. Lett. 20 (1995) 671.

[28] J.Y. Lee, H.-W. Lee, J.W. Kim, Y.S. Yoo and J.W. Hahn, Measurement of ultralow supermirror birefringence by use of the polarimetric differential cavity ringdown technic, Appl. Opt. 39 (2000) 1941.

[29] F. Brandi et al., Measurement of the phase anisotropy of very high reflectivity interferential mirrors, Appl. Phys. B 65 (1997) 351.

[30] K. Long and K.P. Schüler, Polarisation measurement on $\mathrm{e}^{ \pm}$beams, Nucl. Instr. and Meth. A 494 (2002) 75-80.

[31] K.P. Schüler, Polarimeter Studies for TESLA, in Proceedings of the Linear Collider Workshop 2000, Fermilab, Batavia, IL U.S.A. (2001).

[32] G. Moortgat-Pick et al., The role of polarized positrons and electrons in revealing fundamental interactions at the Linear Collider, Phys. Rep. 460 (2008) 131-243. 
Preprint typeset in JINST style - HYPER VERSION

LAL 10-44

\title{
Per Mill Level Control of the Circular Polarisation of the Laser Beam for a Fabry-Perot Cavity Polarimeter at HERA
}

\author{
V. Brisson, R. Chiche, M. Jacquet, C. Pascaud, V. Soskov, Z. Zhang, F. Zomer \\ Laboratoire de l'Accélérateur Linéaire, Univ. Paris-Sud et IN2P3/CNRS, \\ Orsay, France \\ E-mail: mjacquetelal.in2p3.fr
}

M. Beckingham, N. Coppola

DESY

Hamburg, Germany

\begin{abstract}
A precise and fast Fabry-Perot cavity polarimeter, installed in the HERA tunnel in the summer of 2003, was used to measure the longitudinal polarisation of the lepton beam. A complete theoretical model has been developed in order to control at the per mill level the degree of circular polarisation of the laser beam. The transport of this quantity within the whole optical setup has also been performed and controlled at the same level of precision. This is the first time that such a precision is achieved in the difficult, hostile and noisy environment of a particle collider.
\end{abstract}

KEYWORDS: Particle collider, Polarimeter, Ellipsometer, Light polarisation, Quarter Wave Plate.

${ }^{*}$ Corresponding author.

${ }^{\dagger}$ Now at Albert-Ludwigs-University Freiburg, Freiburg, Germany.

\#Now at European XFEL GmbH, Hamburg, Germany. 


\section{Contents}

1. Introduction 1

2. Ellipsometer characterisation 2

2.1 General optical setup and ellipsometer components

2.1.1 General optical setup B

2.1.2 Ellipsometer components

2.2 Model of the ellipsometer and $\chi^{2}$ function

2.2.1 Model of the ellipsometer 6

2.2.2 $\chi^{2}$ function 7

2.3 Ellipsometer parameter determination 8

2.3.1 The data calibration samples 8

2.3.2 Correlation between $e_{\mathrm{QWP}}$ and $S_{3}$

2.3 .3 Results 10

3. Regular measurements of $S_{3}$ and systematics studies 10

3.1 Measurements and systematic uncertainty from the ellipsometer 10

3.2 Transport of $S_{3}$ from ellipsometer to cavity center 12

3.2.1 Parasitic ellipticity from cavity center to ellipsometer entrance 12

3.2.2 Exit transfer matrix $M_{T}$

$3.2 .3 \quad S_{3}$ at the exit of the cavity 13

3.3 Overall $S_{3}$ uncertainty 14

๑. Coherence of $S_{3}$ along the whole optical system 14

4.1 Determination of $M_{E}$

4.2 Coherence of $S_{3}$

5. Summary 17

\section{Introduction}

In a companion article [1] we describe a Compton polarimeter installed at HERA using a FabryPerot resonator to enhance the laser beam power. A relative statistical precision of $2 \%$ per bunch and per minute was achieved on the longitudinal polarisation $P_{z}$ measurement of the electron beam with an estimated relative systematic uncertainty of about $1 \%$. One of the systematic error sources is related to the determination of the degree of circular polarisation $S_{3}$ of the laser beam at the electron-laser interaction point (IP). Since only the product $S_{3} P_{z}$ is determined in Compton polarimetry (see Eq.(5) in [1]), we have to measure precisely $S_{3}$ in order to achieve the same level 
of precision for $P_{z}$. The uncertainty on the $S_{3}$ determination comes mainly from two sources: (1) the measurement of $S_{3}$ itself performed with an ellipsometer usually located close to, but outside, the electron beam pipe and, (2) the transport of the measured $S_{3}$ value through optical elements and vacuum window up to the electron-laser IP. The purpose of the present article is to describe the experimental setup and methods that we have used to reach a few per-mill level of systematic uncertainty on $S_{3}$.

The key component of the experimental setup [1] is an ellipsometer similar to those of the SLAC [2, 3] and Jlab [4, 5] Compton polarimeters. It is composed of a quarter wave plate (QWP), a linear polariser and various photo-detectors. Since an optical model is needed to reconstruct $S_{3}$ from the photometric measurements performed after the polariser, the model accuracy has to be controlled below the per-mill level. The QWP is a crucial component of the ellipsometer. It is usually anti-reflection coated with double layers and thus taken as a simple delay plate in basic optical mo- dels [6]. However, the reflectance of such coated plates is typically of the order of $0.5 \%$, thus limiting the model accuracy to the same level. In order to decrease the model uncertainty, we followed the work of [阿] by choosing an uncoated quartz QWP of high optical quality. In doing so we have to account for multiple reflections inside the anisotropic uniaxial QWP, to model the plate defects and the experimental misalignments, and to perform a fine calibration of the plate thickness. The implementation of an uncoated QWP in the ellipsometer together with a thorough investigation of theoretical models and detector effects within an accelerator environment has never been reported previously and is one of the main topics of this article.

In order to control the transport of accurately measured $S_{3}$ up to the IP, we follow the method developed for the polarimeter at SLAC [8] by modeling the optical elements located between the IP and the ellipsometer, and use optical theorems demonstrated by Jones [9]. In an accelerator this transport is an important issue since optical elements are always present between the IP and the ellipsometer. All birefringence biases from these elements are therefore studied and/or modeled to conserve the required precision on $S_{3}$ at the IP. We also perform a polarisation transport study from the IP up to the laser head by using the optical theorems in [10]. The implementation of these methods is the second topic of this article. To our knowledge they have not been applied in the context of accelerators to the level of accuracy presented here.

The ellipsometer and the characterisation of its optical components are described in Sect. 2. In Sect. 3, the determination of $S_{3}$ with the ellipsometer and the transport from the ellipsometer to the electron-laser IP are described. Finally, in Sect. 4 , the characterisation of the entrance optical line is performed in order to study the coherence of $S_{3}$ along the whole optical system and in particular the coherence of $S_{3}$ at the entrance and at the exit of the Fabry-Perot cavity.

\section{Ellipsometer characterisation}

In this section, the general optical setup and the characterisation of the ellipsometer are described. The principle of an ellipsometer is to send a light beam, of any unknown polarisation, through a QWP. By rotating the plate, the polarisation state of the light is modified and the state at the exit of the plate depends on the state at the entrance. A polariser (Wollaston prism) placed behind the plate spatially separates the beam into two orthogonal linearly polarised states. The analysis of the 
intensities of these two beams in photo-detectors, for various azimuthal angles of the QWP, allows the deduction of the polarisation of the incident beam.

\subsection{General optical setup and ellipsometer components}

\subsubsection{General optical setup}

A schematic overview of the Fabry-Perot cavity optical setup is presented in Fig. 1. A Nd:YAG laser beam of $1064 \mathrm{~nm}$ wavelength passes first through a Glan-Thomson prism in order to provide a purely linearly polarised state and then through an entrance QWP, noted QWP ent This is mounted at the center of a motorised rotating stage to adjust the azimuthal angle $\phi_{\mathrm{ent}}$ and thus to provide an elliptical polarised state. The Glan allows also the beam to pass and to go back after its reflection by the cavity entrance mirror. The reflected beam is analysed in a photodiode, $\mathrm{pd}_{\mathrm{ent}}$, first to determine the azimuthal angles of the plate $\mathrm{QWP}_{\text {ent }}$ for which the light is circularly polarised and secondly to conduct a study on the entrance optical line (Sect. 4.1). The beam then passes through the entrance optics which is composed of a glass plate and two lenses. The glass plate is used to pick up a fraction of the beam for the locking procedure exploiting the "Pound-Drever" technique [11], and the lenses are used to match the laser beam to the cavity fundamental mode. The beam is then precisely aligned with four mirrors (of which two are motorised) before entering a two meter long cavity.

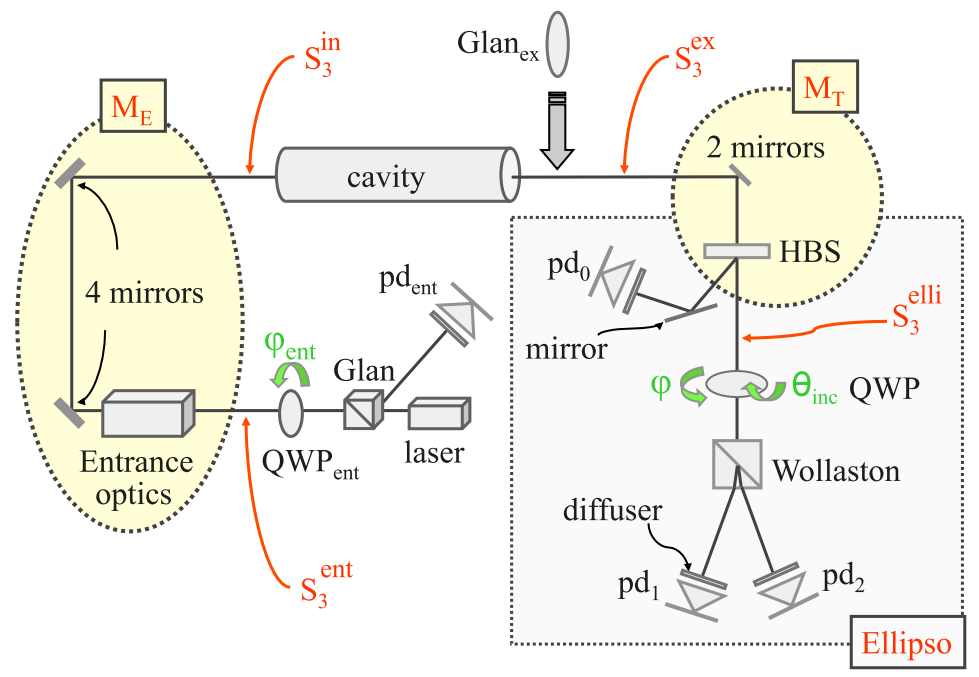

Figure 1. A schematic view of the Fabry-Perot cavity optical system installed in the HERA tunnel. The box "entrance optics" is composed of a glass plate and two lenses. The location of four determination points

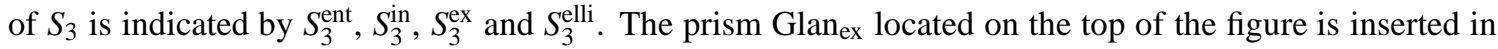
the optical line only for a dedicated study described in Sect. 3.2.2. $M_{E}$ and $M_{T}$ are two transfer matrices discussed in Sects. 3 and 4 .

At the exit of the cavity, the beam is guided with two mirrors to enter the ellipsometer, labeled "Ellipso", which is mainly composed of a QWP followed by a Wollaston prism. The beam passes first through a holographic beam sampler (HBS) in order to extract a small fraction of the entrance 
power (about $1 \%$ of the incident beam). This fraction of the beam is measured in photodiode $\mathrm{pd}_{0}$ and is used as a reference intensity to compensate the effects due to possible laser power variations. The main beam emerging from the HBS enters the QWP. The QWP is mounted at the center of a motorised rotating stage to adjust the azimuthal angle $\phi$. Two screws allow the alignment between the plate and the rotating stage to be adjusted. The rotating stage itself is mounted on a two-axis horizontal stage, a vertical translation stage and an angle-tilting stage to position the beam impact point at the mount center of the rotating stage and to adjust the incident angle $\theta_{\text {inc }}$ between the laser beam and the plate normal direction. The linear and tilted stages are manually controlled with micrometric screws. The Wollaston prism separates the laser beam spatially into two linearly and orthogonally polarised components, and the two transmitted beams from the Wollaston are detected in photodiodes $\mathrm{pd}_{1}$ and $\mathrm{pd}_{2}$. Diffusers are placed in front of each photodiode in order to adjust the power entering the photodiodes.

The ellipsometer is used to measure the circular polarisation $S_{3}$ of the laser beam of any unknown polarisation by varying the azimuthal angle of the plate and analysing the intensities emerging from the prism.

\subsubsection{Ellipsometer components}

The HBS: The beam sampler is a hologram in engraved relief on a transparent substrate (silica) inducing forward diffraction. The birefringence of the HBS alone has been measured before its installation in the cavity system and the result is compatible with zero [12].

The Wollaston prism: The most important characteristic of the prism is its extinction rate, which is less than a few $10^{-5}$ as given by the manufacturer and has been confirmed experimentally.

The quarter wave plates: As will be described in Sect. 2.3, two QWPs $\mathrm{pl}_{1}$ and $\mathrm{pl}_{2}$ with different nominal thicknesses of $e_{\text {nom }}^{(1)}=91.2 \mu \mathrm{m}$ and $e_{\text {nom }}^{(2)}=639.9 \mu \mathrm{m}$ are used in the ellipsometer for its characterisation in order to increase the constraints of the system. Each QWP is a parallel plate of high quality quartz manufactured especially for the ellipsometer characterisation purpose, and has a delay tolerance of $1 / 300$ and a thickness tolerance of a few micrometers with a parallelism between the two faces of the order of 10 seconds of arc. The optical axis is contained in the plane of the plate.

The detection system: The detection system consists of three photodiodes $\mathrm{pd}_{0}, \mathrm{pd}_{1}$ and $\mathrm{pd}_{2}$ made of a gallium arsenide and indium alloy (InGaAs). Each photodiode and their electronics are thermalised with a Peltier module to prevent temperature variations which might be of a few degrees in the HERA environment and could induce a variation in the photodiode readout larger than the required precision. Using Peltier modules, a stability level of tenth of a degree is achieved. The photodiodes are read out with 12-bit analog-to-digital converters at a maximum sample rate of $2 \mathrm{MHz}$. For each measurement, the mean value over ten thousand signals $I_{\mathrm{pd}}$ is recorded after subtraction of pedestal Ped for each photodiode. The effect of laser power variations is taken into account by normalising the measurement to that of the reference photodiode $\mathrm{pd}_{0}$. Explicitly, pho- 
todiode intensities $I_{1}$ and $I_{2}$ used in the following can be written as:

$$
I_{1,2}=\frac{\left\langle I_{\mathrm{pd}_{1,2}}\right\rangle_{10000}-\mathrm{Ped}_{1,2}}{\left\langle I_{\mathrm{pd}_{0}}\right\rangle_{10000}-\mathrm{Ped}_{0}}
$$

A study of photodiodes in an optical laboratory has shown that in such a clean environment, the temperature regulation, the subtraction of photodiode pedestals and the laser power variation effect can be controlled such that $I_{1}$ and $I_{2}$ are known at the per-mill level. The HERA accelerator environment is more noisy; effects such as larger temperature variations and the presence of quadrupoles and dipoles, the synchrotron radiation, accelerating cavities and long cables affect the precision of the photodiode measurements. This is illustrated in Figs. 2(a) and (b) where the distributions of $I_{2}$ from two data samples recorded in the HERA tunnel at different moments are shown. Each entry in the histograms is a measurement of $I_{2}$ as defined in Eq.(2.1) and the duration of data taking for each sample was approximately fifteen minutes. Fig. (2) (a) represents a well clustered distribution whereas Fig. 2(b) shows two populations. To take into account this kind of drift, the corresponding
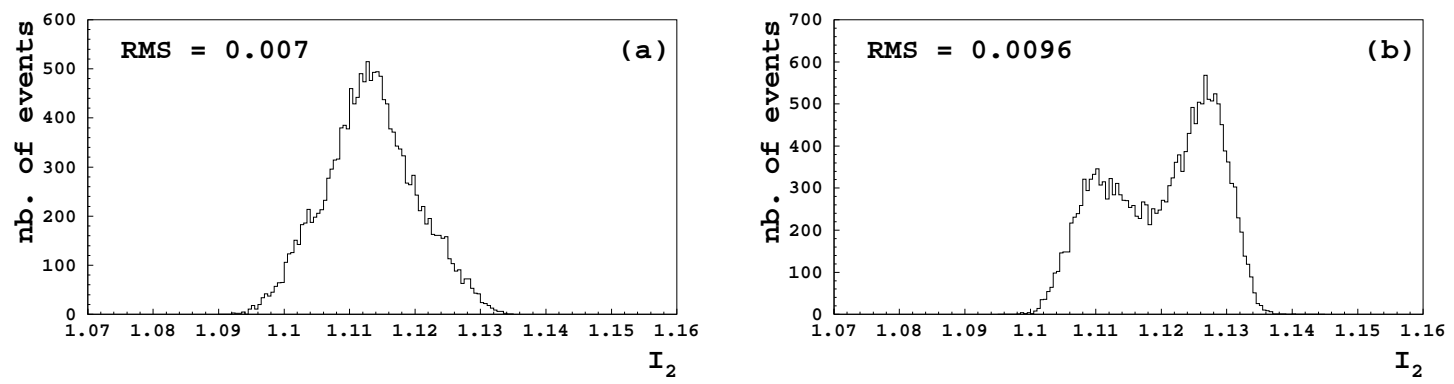

Figure 2. Histograms of $I_{2}$ as defined in Eq.(2.1) for two data sets (a) and (b) taken during fifteen minutes each at different moments.

error $\sigma_{I_{1,2}}$ is defined as the root mean square (RMS) value of the histogram, whether it has a Gaussian shape or not. In this way, the unknown long term effects such as those illustrated in Fig. 2(b) are included in the measurement errors. The errors are then parameterised as a function of the intensities as:

$$
\sigma_{I_{1,2}=A_{1,2}} I_{1,2}+B_{1,2}
$$

Fig. 3 shows the errors $\sigma_{I_{1}}$ (a) and $\sigma_{I_{2}}$ (b) as a function of $I_{1}$ and $I_{2}$ respectively and the corresponding parameterisation of Eq.(2.2), for data sets recorded at various azimuthal angles of the ellipsometer QWP in order to cover the entire range of intensity values. These errors may depend on the duration of acquisition time which will be varied for systematics studies (Sect. 3.1).

The accurate measurement of the degree of circular polarisation $S_{3}$ of the laser beam requires a precise control and characterisation of this ellipsometer. Therefore before determining $S_{3}$, the ellipsometer will be first used as a calibration system to characterise precisely some of its optical components. For this, a complete simulation model has been developed. 

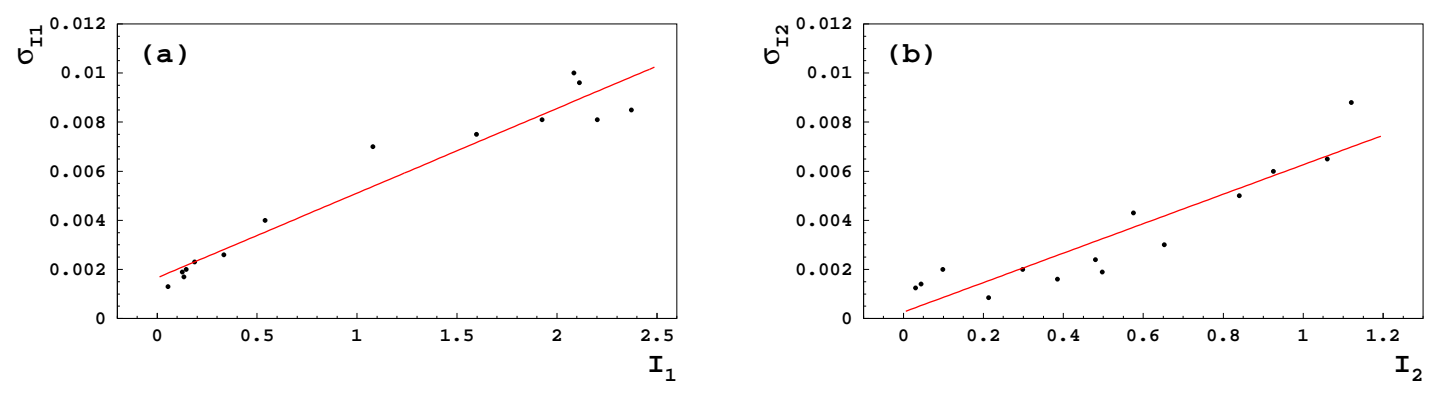

Figure 3. Errors $\sigma_{I_{1}}$ (a) and $\sigma_{I_{2}}$ (b) as a function of $I_{1}$ and $I_{2}$, respectively. The lines correspond to straight line fits to the measured errors according to Eq.(2.2).

\subsection{Model of the ellipsometer and $\chi^{2}$ function}

\subsubsection{Model of the ellipsometer}

The polarisation state $\mathbf{E}_{\text {elli }}$ at the entrance of the ellipsometer and the associated degree of circular polarisation $S_{3}^{\text {elli }}$ can be written in the most general form as functions of two angles $\xi_{\text {elli }}$ and $\phi_{\text {elli }}$ as [13]:

$$
\mathbf{E}_{\text {elli }}=\left(\begin{array}{l}
\cos \xi_{\text {elli }} \\
\sin \xi_{\text {elli }} e^{i \phi_{\text {elli }}}
\end{array}\right), \quad S_{3}^{\text {elli }}=2 \cos \xi_{\text {elli }} \sin \xi_{\text {elli }} \sin \phi_{\text {elli }}
$$

The expression of the two transmitted fields $\mathbf{E}_{1}$ and $\mathbf{E}_{2}$ emerging from the Wollaston prism and the associated theoretical intensities $T_{1}$ and $T_{2}$ can then be written as:

$$
\mathbf{E}_{1(2)}=\mathscr{M}_{1(2)}^{\text {elli }} \mathbf{E}_{\text {elli }}, \quad T_{1(2)}=\left|\mathbf{E}_{1(2)}\right|^{2},
$$

where $\mathscr{M}_{1}^{\text {elli }}$ and $\mathscr{M}_{2}^{\text {elli }}$, standing for the corresponding Jones transmission matrices [14], are computed from a theoretical model as follows:

The Wollaston cube: Two small parameters $\varepsilon_{x}$ and $\varepsilon_{y}$ are introduced to take into account in the Wollaston Jones matrix a possible small birefringence along its two optical axes [15].

The quarter wave plate: The model used for the QWP takes into account the multiple reflections of the wave inside a quartz plate of indices $n_{o}$ and $n_{e}$, of thickness $e_{\mathrm{QWP}}$, and whose optical axis has an angle $\phi_{\mathrm{oa}}$ with respect to the laboratory frame. This angle has two components: $\phi_{\mathrm{oa}}=\phi+\phi_{0}$, where $\phi$ is the azimuthal angle of the QWP motorised rotating stage and $\phi_{0}$ is an azimuthal reference angle reflecting the fact that the orientation of the optical axis in the plane of the plate is not a priori at position $\phi=0$. The simulation also models the passage of a light wave through the plate at a non zero incident angle $\theta_{\text {inc }}$, which is equal to the number of tilting stage screw turns $\left(X_{\text {turn }}\right)$ times the tilt angle value of one screw turn $\left(\theta_{\text {turn }}\right)$. The light beam is treated as a plane wave since, at small incident angles (i.e. less than $0.1 \mathrm{rad}$ for the work described here), the comparison between a plane wave treatment and a Gaussian wave treatment shows that the Gaussian character of the wave can be neglected [16]. Also, the contribution of the optical activity of the crystal [17] as well as the surface roughness [18] has been studied and found to be negligible (a relative contribution of less than $10^{-6}$ ). 
Misalignments: A realistic description of the optical system must also take into account the following experimental misalignments:

- A misalignment of the QWP plane with respect to the Wollaston prism axes, which is modeled by a small tilt angle $\delta_{W}$ of the normal to the QWP.

- A misalignment due to the fact that the laser beam may not enter the QWP exactly at the plate center of the mount: in that case, because the two plate surfaces may not be perfectly parallel, the thickness crossed by the light can vary during an azimuthal rotation of the plate. To take into account this effect, the plate is modeled by a variable thickness as: $e=e_{\mathrm{QWP}}[1+$ $\left.\left(\delta_{c} / e_{\mathrm{QWP}}\right) \cos \left(\phi-\phi_{c}\right)\right]$ where $e_{\mathrm{QWP}}$ is the thickness of the plate in case of a perfect alignment situation, $\delta_{c}$ represents a small shift between the laser beam impact point and the plate center of the mount, and $\phi_{c}$ is an arbitrary reference azimuthal angle, as the position $\phi=0$ of the QWP may not be the position which maximises the plate thickness $e$. One new parameter $\delta_{c}$ is introduced each time the incident angle $\theta_{\text {inc }}$ changes.

According to this model of ellipsometer components and misalignments, for a given ellipsometer QWP plate $\mathrm{pl}_{k}$ of thickness $e_{\mathrm{QWP}}^{k}$, a given incident angle $\theta_{\mathrm{inc}}^{j}$, azimuthal angle $\phi^{i}$ of the plate, and polarisation state $\mathbf{E}_{\text {elli }}^{\ell}$, the two theoretical intensities $\mathrm{T}_{1(2)}$ of Eq.(2.4) can be written as functions of ellipsometer parameters as:

$$
\begin{array}{r}
T_{1(2)}^{i j k \ell} \equiv f_{1(2)}\left(n_{o}, n_{e}, \varepsilon_{x}, \varepsilon_{y}, e_{\mathrm{QWP}}^{k}, \theta_{\mathrm{turn}} \times X_{\mathrm{turn}}^{j},\right. \\
\left.\phi_{0}^{k}+\phi^{i}, \xi_{\mathrm{elli}}^{\ell}, \phi_{\mathrm{elli}}^{\ell}, \delta_{W}^{k}, \delta_{c}^{j}, \phi_{c}^{k}\right) .
\end{array}
$$

All the details of the model described here can be found in [19] where the modeling and the calculations relative to the Wollaston cube, the QWP and the optical misalignments are explicitly given. In particular, the calculation of the Jones transmission matrix of a quartz plate at a non zero incident angle, with internal reflections being taken into account, is explicitly performed in the appendix of [19]. Simulations show that multiple reflections, plate defects or optical misalignments contribute at the few percent level to the transmitted intensities $T_{1(2)}$. Achieving the per-mill level for $S_{3}$ measurement is therefore only possible using this complete model description.

\subsection{2 $\chi^{2}$ function}

To characterise the ellipsometer and distinguish effects due to optical misalignments from those due to plate defects or those due to the light polarisation state, the principle is to record ellipsometer experimental data $I_{1(2)}$ (as defined in Eq. 2.1) ) and to minimise the following $\chi^{2}$ function:

$$
\begin{aligned}
\chi^{2}= & \sum_{k=1, N_{\mathrm{pl}}} \sum_{\ell=1, P_{k}} \sum_{j=1, \Theta_{k}} \sum_{i=1, N_{\phi}} \\
& {\left[\left(\frac{R_{1}^{j \ell k} T_{1}^{i j \ell k}-I_{1}^{i j \ell k}}{\sigma_{1}^{i j \ell k}}\right)^{2}+\left(\frac{R_{2}^{j \ell k} T_{2}^{i j \ell k}-I_{2}^{i j \ell k}}{\sigma_{2}^{i j \ell k}}\right)^{2}\right], }
\end{aligned}
$$

where $N_{\mathrm{pl}}$ refers to the number of the QWP used in the data taking, $P_{k}$ to the number of polarisation states, $\Theta_{k}$ to the number of incident angles and $N_{\phi}$ to the number of azimuthal angles of the QWP.

$T_{1(2)}^{i j \ell k}\left(I_{1(2)}^{i j \ell k}\right)$ is the theoretical (experimental) photodiode intensity calculated (measured) when the 
plate $\mathrm{pl}_{k}$ is inserted in the ellipsometer, at the $\ell^{\text {th }}$ polarisation state $\mathbf{E}_{\text {elli }}$, the $j^{\text {th }}$ value of $\theta_{\text {inc }}$ and the $i^{\text {th }}$ value of $\phi . \sigma_{1(2)}^{i j \ell k}$ is the uncertainty of $I_{1(2)}^{i j \ell k}$ in Eq.(2.2). $R_{1(2)}^{j \ell k}$ are normalisation factors which are easily determined by solving $\partial \chi^{2} / \partial R=0$. The minimisation of the $\chi^{2}$ (Eq.(2.6) leads to parameter values of Eq.(2.5), excepted for $X_{\text {turn }}^{j}, \phi^{i}$ and $n_{e} . X_{\text {turn }}^{j}$ and $\phi^{i}$ take some known values of the tilting and rotating stages, respectively. The extraordinary index $n_{e}$ is fixed because our constraints are not sufficient to determine $n_{o}$ and $n_{e}$ at the same time, and so the quantity that is determined in practice is the birefringence $n_{e}-n_{o}$. The quartz indices being measured at a few $10^{-5}$ level by previous studies [20, 21, 22], the determination of the birefringence by the ellipsometer provides thus a good test of the validity of the model.

\subsection{Ellipsometer parameter determination}

The determination of ellipsometer parameters (Eq.(2.5) has been performed twice, independently in two different environments corresponding to the optical laboratory and the HERA tunnel, with two different data taking procedures.

The first data sample recorded in the optical laboratory is devoted to determine all ellipsometer characteristics and in particular the thickness of the QWP and the index $n_{o}$. The determination of both $e_{\mathrm{QWP}}$ and $n_{o}$ requires a long and meticulous data taking procedure since it turns out that the solution of the $\chi^{2}$ minimisation is not unique: several combinations $\left(e_{\mathrm{QWP}}, n_{o}\right)$ can minimise the $\chi^{2}$. In order to resolve such an ambiguity, two uncoated plates $\mathrm{pl}_{1}$ and $\mathrm{pl}_{2}$ with different thicknesses are used by inserting one after the other in the ellipsometer. In addition, for each plate, several data sets are taken for different incident angles.

The second data sample was recorded after the installation of the setup in the HERA tunnel in order to characterise again the system, since all the optical components were dismounted to be transported from the optical laboratory to the tunnel. In the tunnel, data taking conditions were much more difficult than in the optical laboratory, and in particular, the tunnel accesses were limited to a few hours per month. The corresponding data taking procedure thus has to be simpler. To avoid ambiguous solutions on the thickness of plate $\mathrm{pl}_{1}$, its fit range is restricted to around the expected value obtained from the laboratory calibration.

\subsubsection{The data calibration samples}

The first calibration of the ellipsometer was performed in the clean optical laboratory, where the room temperature was regulated at $25^{\circ}$. For a given laser beam polarisation state, ellipsometer measurements were recorded for each plate $\mathrm{pl}_{1}$ and $\mathrm{pl}_{2}$ at various incident angles $\theta_{\text {inc }}$ between the laser beam and the plate normal direction, and, for each $\theta_{\text {inc }}$, the QWP was turned azimuthally of an angle $\phi$ in step of $1^{\circ}$ from $0^{\circ}$ to $360^{\circ}$. A Monte Carlo study shows that to provide enough constraints to minimise the $\chi^{2}$ and determine all the ellipsometer parameters of Eq.(2.5), data have to be recorded at four (two) different values of $\theta_{\text {inc }}$ for the QWP $\mathrm{pl}_{1}\left(\mathrm{pl}_{2}\right)$, and it is sufficient to have only one polarisation state of a given $\left(\xi_{\text {elli }}, \phi_{\text {elli }}\right)$. Each time the incident angle $\theta_{\text {inc }}$ was changed, a long procedure was applied to displace manually the plate transversally with the linear stage micrometric screws in order to recover precisely the matching of the plate mechanical center with the laser impact point. The six samples recorded at different values of $\theta_{\text {inc }}$ containing 360 entries each are called $D_{d}^{\text {lab }}(d=1, \cdots, 6)$ and, when introduced in the $\chi^{2}$ function, values of subscripts in Eq.(2.6) are $N_{\mathrm{pl}}=2, P_{1}=1, P_{2}=1, \Theta_{1}=4, \Theta_{2}=2$ and $N_{\phi}=360$. 
The second data set was recorded in the HERA tunnel where the room temperature was regulated at $35^{\circ}$. In order to control the data taking procedure from outside tunnel to be independent of the short duration of tunnel access, only one plate $\left(\mathrm{pl}_{1}\right)$ was used in the ellipsometer and the incident angle $\theta_{\text {inc }}$ between the light beam and this QWP remained fixed and equal to zero. In this configuration, the $\chi^{2}$ minimisation was performed by using three recorded data samples, each with a different azimuthal angle of the entrance plate $\mathrm{QWP}_{\text {ent }}$. In this way, the light entering the ellipsometer has three different polarisation states. For each of these three $\mathrm{QWP}_{\text {ent }}$ azimuthal positions, the ellipsometer QWP was also turned azimuthally through an angle $\phi$ in step of $1^{\circ}$ from $0^{\circ}$ to $360^{\circ}$. These three data samples are called $D_{d}^{\mathrm{HERA}}(d=1, \cdots, 3)$ and the corresponding values for the superscripts in Eq.(2.6) are: $N_{\mathrm{pl}}=1, P_{1}=3, \Theta_{1}=1$ and $N_{\phi}=360$.

\subsubsection{Correlation between $e_{\mathrm{QWP}}$ and $S_{3}$}

Among all ellipsometer parameters, the dominant source of systematic error on $S_{3}$ comes from the QWP thickness uncertainty. Thus, before giving the results of the minimisations using the two sets of data samples previously described, it is interesting to show the correlation between the plate thickness $e_{\mathrm{QWP}}$ and $S_{3}$. The effect on the $S_{3}$ determination is estimated by simulating an ellipsometer data sample with a degree of circular polarisation $S_{3}^{\text {true }}$ and a plate thickness $e_{\text {gen }}$. Using this sample, various minimisations of the $\chi^{2}$ are performed by letting only $S_{3}^{\text {elli }}$ free (i.e. only the two angles $\xi_{\text {elli }}$ and $\phi_{\text {elli }}$ ), by fixing the plate thickness to different values $e_{\text {fix }}$ slightly different from $e_{\text {gen }}$, and by fixing all the other parameters to their generated values. The quantity $\left|\left(S_{3}^{\text {true }}-S_{3}^{\text {elli }}\right) / S_{3}^{\text {true }}\right|$ presented in Fig. $⿴$ as a function of $e_{\text {fix }}-e_{\text {gen }}$ shows that a systematic error of one micometer on the plate thickness leads to a systematic error around $0.5 \%$ on $S_{3}$. A precise knowledge of the plate thickness inside the thickness tolerance of a few micrometers given by the manufacturer has therefore to be reached to keep a systematic error at the per-mill level on the measurement of $S_{3}$. Achieving this precision is only possible using the complete model described previously.

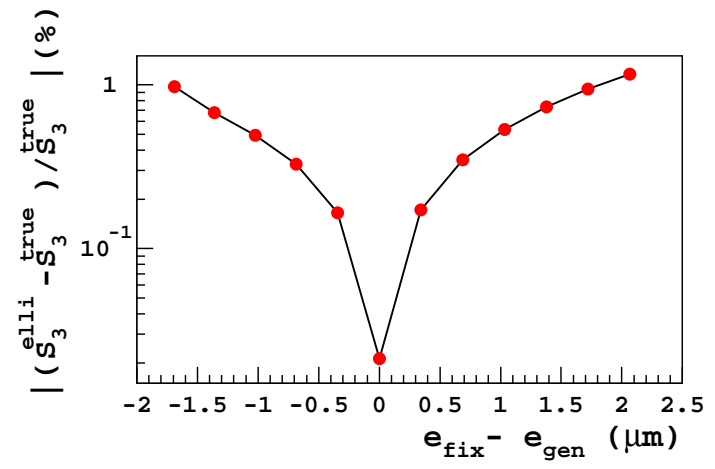

Figure 4. Relative error on $S_{3}^{\text {elli }}$ as a function of the uncertainty on the ellipsometer QWP thickness. 


\subsubsection{Results}

The $\chi^{2}$ minimisation procedure was tested first by using simulated samples generated with Eq.(2.4) following closely the experimental data. It was found that all fitted parameters were in agreement with the generated ones within a precision of a few per-mill. The minimisation is then performed independently with the two experimental data sets $D_{d}^{\mathrm{lab}}$ and $D_{d}^{\mathrm{HERA}}$ and leads to values of $\chi^{2}$ per degree of freedom equal to 1.07 and 2.09 respectively. The excellent agreement between experimental intensities and theoretical ones based on the fit is illustrated by a typical example in Fig. 5, where the quantities $I_{1,2}$ and $R_{1,2} T_{1,2}$ are presented for the sample $D_{1}^{\mathrm{HERA}}$ as a function of the azimuthal angle $\phi$ of the ellipsometer QWP.
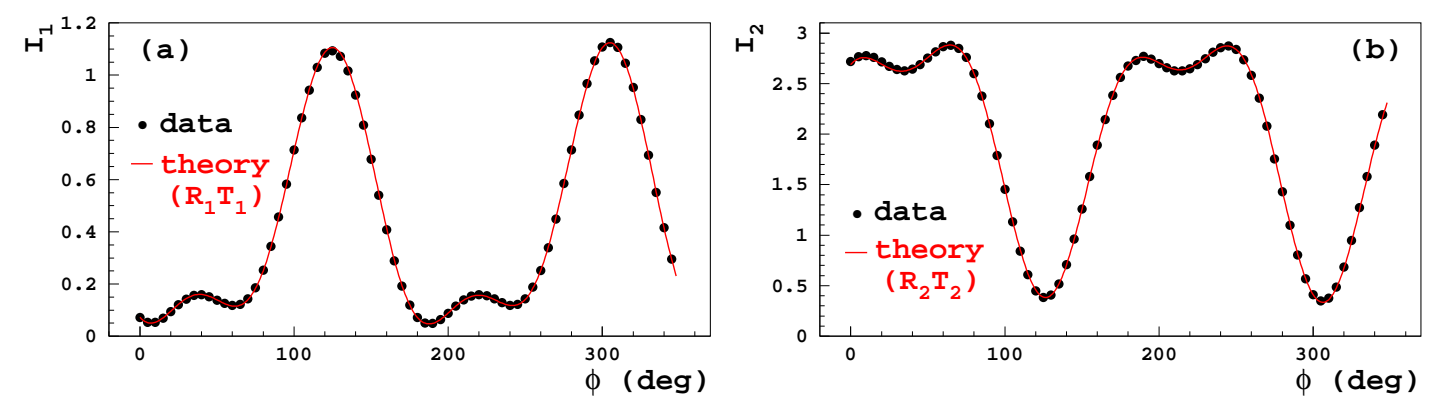

Figure 5. Experimental intensities (black bullets, for clarity only a subsample is shown) $I_{1}$ (a) and $I_{2}$ (b) compared with theoretical ones (curves) derived from the fit, as a function of the azimuthal angle $\phi$ of the ellipsometer QWP, for the data file $D_{1}^{\mathrm{HERA}}$.

All ellipsometer parameters determined from the two minimisations using the data sets $D_{d}^{\text {lab }}$ or $D_{d}^{\mathrm{HERA}}$ are found to be realistic and well defined. Among them, one interesting quantity is the quartz birefringence value $n_{e}-n_{o}$ which can be compared with textbook values previously determined. In [21, 22], birefringence measurements were performed at a temperature of $18^{\circ}$ and $22^{\circ}$. Our measurements in the optical laboratory and in the tunnel were carried out at higher temperatures of $25^{\circ}$ and $35^{\circ}$, respectively. Based on the relation of optical index variation with temperature [23], quartz birefringence values of [21, 22] are scaled to $T=25^{\circ}$ and at $T=35^{\circ}$ and are shown in Fig. 6 together with the two birefringence values obtained from the fits. The two fit values agree at better than one per-mill with the ones quoted in the references.

As for the birefringence, all other results determined in the optical laboratory are compared with those in the tunnel and good agreement are found once the effects of temperature difference and optical alignment difference are taken into account [19].

\section{Regular measurements of $S_{3}$ and systematics studies}

\subsection{Measurements and systematic uncertainty from the ellipsometer}

During the data taking period of the cavity polarimeter, when the cavity was locked in resonant state and the azimuthal angle $\phi_{\text {ent }}$ of the entrance QWP was such that the light is close to a fully right or left circularly polarised state $S_{3}= \pm 1$ [1], values of $S_{3}^{\text {elli }}$ were regularly determined. 


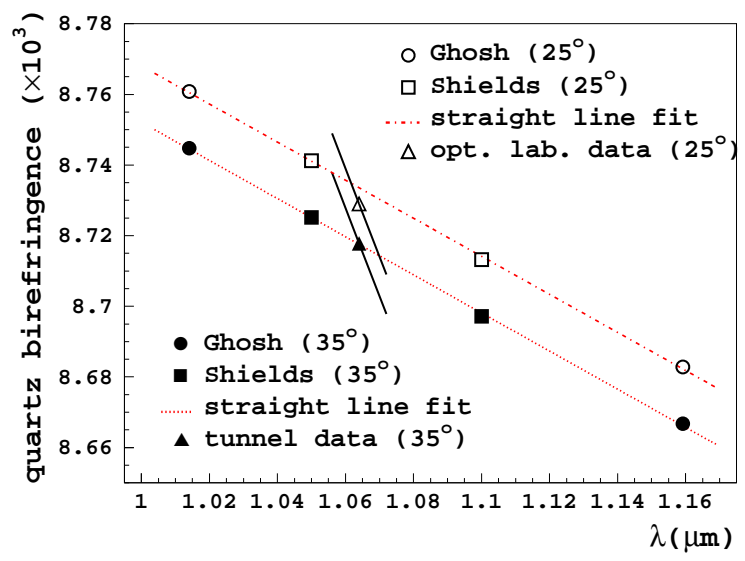

Figure 6. Quartz birefringence values scaled to $25^{\circ}$ and $35^{\circ}$ from textbook values [21, 22], as a function of the wavelength. Dashed lines are straight line fits to four scaled textbook points. Birefringence values determined from the fits to data $D^{\text {lab }}$ and $D^{\text {HERA }}$ are indicated by an open and a solid triangle, respectively.

Each value of $S_{3}^{\text {elli }}$ is extracted from a data sample recorded with the ellipsometer and containing 180 photodiode signals (as defined in Eq.(2.1)) corresponding to a $2^{\circ}$-step azimuthal turn of the ellipsometer QWP. The duration of data taking was about ten minutes for each sample.

To extract $S_{3}^{\text {elli }}$ and its uncertainty $\sigma_{S_{3}^{\text {elli }}}$, the $\chi^{2}$ function defined in Eq.2.6) is minimised. The only fitted parameters are the polarisation state parameters $\xi_{\text {elli }}$ and $\phi_{\text {elli }}$ and all other parameters are fixed to values previously determined by the characterisation of the tunnel optical system as described in Sect. 2. Thanks to a heat regulation system, the temperature inside the isotherm house (see [1]) was controlled within $\pm 0.3^{\circ} \mathrm{C}$ which ensured a perfect stability of the optical axis (defined by the positions of the cavity mirrors) over time and therefore the stability of our ellipsometer calibration.

The uncertainty $\sigma_{S_{3}}$ oli obtained from the $\chi^{2}$ minimisation is of the order of a few $10^{-4}$. During one year (from June 2006 to June 2007), the azimuthal positions of the plate QWP $_{\text {ent }}$ defining a left or right circularly polarised laser beam were changed only three times, either after an hardware problem on the rotating mount controller of the plate, or after a dedicated check involving the plate or the photodiode $\mathrm{pd}_{\text {ent }}$. The $S_{3}^{\text {elli }}$ measurements were very stable over time since, over the one-year period, they have shown a stability of a few per-mill [19].

Given the precise ellipsometer calibration procedure described in Sect. 2.2, the only remaining source of systematic uncertainty on $S_{3}^{\text {elli }}$ concerns the duration of the data taking. Indeed, the duration of one measurement sample taken with the ellipsometer can have an effect on the photodiode intensity distributions because of long term fluctuation as shown in Fig. 2(b). This duration depends on the chosen interval between two consecutive azimuthal angles $\phi$ of the ellipsometer QWP. To study this effect, the entrance plate $\mathrm{QWP}_{\text {ent }}$ was fixed to a given azimuthal position and several measurement samples were recorded using the ellipsometer for different durations ranging from three to twenty minutes. The extracted $S_{3}^{\text {elli }}$ values from these samples are found to be compatible within two per-mill [19]. To be conservative, an error of three per-mill is quoted for the 
uncertainty due to the duration of data taking.

\subsection{Transport of $S_{3}$ from ellipsometer to cavity center}

The precise $S_{3}$ values obtained above concern the degree of circular polarisation at the entrance of the ellipsometer. What we are interested in is, however, the $S_{3}$ value at the electron-laser interaction point, i.e. at the center of the Fabry-Perot cavity. A priori, these two values are the same, but because of the presence of optical components between the two, a small difference could be induced. The transport of $S_{3}$ and its uncertainty are the subject of this section.

\subsubsection{Parasitic ellipticity from cavity center to ellipsometer entrance}

Between the center of the cavity and the entrance of the ellipsometer (see Fig. 1), the exit mirror and the exit window of the Fabry-Perot cavity and the optical system $M_{T}$ could be a source of birefringence and may induce parasitic ellipticity, modifying the laser beam polarisation. The birefringence of the substrate, the coating and the mounting system of the exit mirror and the exit window has been estimated or measured from dedicated studies [24, 19]. It was shown that the bias induced on $S_{3}$ from the center to the exit of the cavity is at the utmost of the order of $3 \times 10^{-5}$. The remaining dominant source of parasitic ellipticity is associated to the $45^{\circ}$ dielectric mirrors used to guide the light into the ellipsometer because of their different reflection coefficients for two electric field components: one in the incident plane and the other perpendicular to it [13, 25]. To determine this ellipticity, the transfer matrix $M_{T}$ of the system has to be determined.

\subsubsection{Exit transfer matrix $M_{T}$}

An optical theorem demonstrated by R. Clark Jones [9] states that any optical system composed of any non-absorbing components may always be replaced by a partial polariser placed between two delay plates, with the addition of a rotator inserted at any position in the system. Under the assumption that no power is absorbed in optical materials, the Jones matrices of a partial polariser, a delay plate and a rotator $P_{p_{1} p_{2}}, G_{\gamma}$ and $R_{\theta}$ can be written as [6]:

$$
P_{p_{1} p_{2}}=\left(\begin{array}{cc}
p_{1} & 0 \\
0 & p_{2}
\end{array}\right), \quad G_{\gamma}=\left(\begin{array}{cc}
e^{i \gamma} & 0 \\
0 & e^{-i \gamma}
\end{array}\right), \quad R_{\theta}=\left(\begin{array}{cr}
\cos \theta & -\sin \theta \\
\sin \theta & \cos \theta
\end{array}\right)
$$

Following the Jones theorem [9] and starting with a completely linear polarisation state $\mathbf{E}_{G}=$ $\left(\cos \phi_{G}, \sin \phi_{G}\right)^{T}$, the theoretical final state $\mathbf{E}_{\text {th }}$ after passing through an optical system of transfer matrix $M_{T}$ can then be modeled as:

$$
\begin{array}{ll} 
& \mathbf{E}_{\text {th }} \equiv\left(\cos \xi^{\text {th }}, \sin \xi^{\text {th }} e^{i \phi^{\text {th }}}\right)^{T}=M_{T} \mathbf{E}_{G} \\
\text { with } & M_{T}=R_{\theta_{1}} G_{\gamma_{1}} R_{\theta_{2}} P_{p_{1} p_{2}} R_{\theta_{3}} G_{\gamma_{2}} .
\end{array}
$$

In order to apply this theorem to determining the matrix $M_{T}$ of the two mirrors and the HBS located at the exit of the Fabry-Perot cavity, a special configuration of the system was set up by

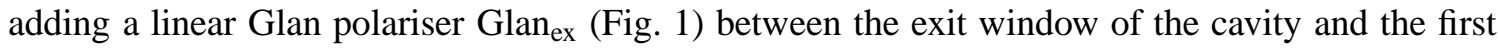
mirror. Such a device polarises the beam in a completely linear state $\mathbf{E}_{G}$ before it enters the optical elements of the exit line. In this configuration, a number of data samples $N_{d}$ were recorded using 
the ellipsometer, each with a different azimuthal angle $\phi_{G}^{\ell}\left(\ell=1, \cdots, N_{d}\right)$ of the polariser Glan $_{\mathrm{ex}}$. For each $\phi_{G}^{\ell}$, the ellipsometer QWP was rotated azimuthally from $0^{\circ}$ to $360^{\circ}$.

A fit to these data samples is performed by minimising the $\chi^{2}$ function defined in Eq.(2.6) to obtain the only free parameters $\xi_{\text {elli }}^{\ell}$ and $\phi_{\text {elli }}^{\ell}\left(\ell=1, \cdots, N_{d}\right)$ of the beam polarisation state after

the HBS. Using the resulting $\xi_{\text {elli }}^{\ell}$ and $\phi_{\text {elli }}^{\ell}$ and their uncertainties $\sigma_{\xi_{\text {elli }}^{\ell}}$ and $\sigma_{\phi_{\text {elli }}^{\ell}}$, the following $\chi^{2}$ function is constructed in order to determine the elements of the matrix $M_{T}$ :

$$
\chi^{2}=\sum_{\ell=1, N_{d}}\left[\left(\frac{\xi_{\text {th }}^{\ell}-\xi_{\text {elli }}^{\ell}}{\sigma_{\xi_{\text {elli }}^{\ell}}}\right)^{2}+\left(\frac{\phi_{\text {th }}^{\ell}-\phi_{\text {elli }}^{\ell}}{\sigma_{\phi_{\text {elli }}^{\ell}}}\right)^{2}\right],
$$

where $\xi_{\mathrm{th}}^{\ell}$ and $\phi_{\mathrm{th}}^{\ell}$ are the theoretical angles defining the $\ell^{\text {th }}$ polarisation state after the HBS. By using Eqs.(3.1), (3.2), these angles can be written as functions of the parameters $\theta_{1}, \theta_{2}, \theta_{3}, \gamma_{1}, \gamma_{2}, p_{1}$ and $p_{2}$ of the matrix $M_{T}$ and of the angle $\phi_{G}^{\ell}$ of the linear initial polarisation state. A Monte Carlo study of the $\chi^{2}$ function defined in Eq. (3.3) shows that some elements of the matrix $M_{T}$ are completely correlated and that the system can be described only with one delay plate, one partial polariser and two rotators. The parameters of $M_{T}$ in Eq.(3.2) are therefore restricted to $\gamma_{1} \equiv \gamma_{T}, p_{1} \equiv p_{T}, p_{2}=1$, $\theta_{3}=0$ and $\gamma_{2}=0$. The minimisation of the $\chi^{2}$ defined in Eq.(3.3) leads to $\gamma_{T}=(13.1 \pm 1.4) \mathrm{mrad}$ and $p_{T}=1.001 \pm 0.001$, thereby showing that the exit optical system behaves like a delay plate inducing a birefringence of the order of a few tens of mrad.

A cross-check of this study has been performed by instead placing the additional polariser Glan $_{\mathrm{ex}}$ between the HBS and the ellipsometer QWP. As previously, ellipsometer data sets were recorded for several azimuthal angles of the polariser. Applying the same procedure, the matrix $M_{T}$ is now expected to be compatible with the identity matrix, since there is no optical component between the Glan and the entrance of the ellipsometer. The result of the fit, with an angle of $(1.5 \pm 4.0) \mathrm{mrad}$ for the delay plate and a value of $1.004 \pm 0.005$ for the partial polariser parameter, constitutes a valuable check of the robustness of our model describing the exit beam line of the Fabry-Perot cavity.

\subsection{3 $S_{3}$ at the exit of the cavity}

Removing the polariser Glan $_{\text {ex }}$ to recover the standard setup of the optical system, the degree of circular polarisation $S_{3}^{\text {ex }}$ at the exit of the cavity has now to be determined as a function of $S_{3}^{\text {elli }}$ at the entrance of the ellipsometer (Sect. 3.1). The polarisation state $\mathbf{E}_{\mathrm{ex}}$ at the exit of the cavity is related to $\mathbf{E}_{\text {elli }}$ (Eq.(2.3) as $\mathbf{E}_{\mathrm{ex}}=M_{T}^{-1} \mathbf{E}_{\mathrm{elli}}$. The development of this field expression leads to the relation:

$$
S_{3}^{e x}=S_{3}^{\text {elli }}+\delta S_{\mathrm{ex}}\left(\theta_{1}, \theta_{2}, \gamma_{T}, p_{T}, \xi_{\mathrm{elli}}, \phi_{\mathrm{elli}}\right) .
$$

The relation (3.4) applied to several values of $S_{3}^{\text {elli }}$ determined by the ellipsometer shows that the correction values $\delta S_{\text {ex }}$ are all below five per-mill. As the HBS is not the cause of this parasitic ellipticity (see Sect. 2.1), the bias is due to the two mirrors system. This confirms a measurement performed at Saclay in 1999 for the TJNAF polarimeter [26] in which the effect of the two mirrors on the determination of $S_{3}$ was measured to be of the order of a few per-mill.

The bias $\delta S_{\text {ex }}$ is calculable for each value of $S_{3}^{\text {elli }}$ and therefore does not enter as a syste- matic error but is explicitly determined to correct $S_{3}^{\text {elli }}$. The uncertainty $\sigma_{S_{3}^{\text {ex }}}$ of $S_{3}^{\text {ex }}$ due to the transfer from 
$S_{3}^{\text {elli }}$ to $S_{3}^{\text {ex }}$ are of order of a few $10^{-4}$ and has been calculated from the $M_{T}$ elements as follows: for four combinations $\left(\gamma_{T}+\sigma_{\gamma_{T}}, p_{T}+\sigma_{p_{T}}\right),\left(\gamma_{T}-\sigma_{\gamma_{T}}, p_{T}+\sigma_{p_{T}}\right),\left(\gamma_{T}+\sigma_{\gamma_{T}}, p_{T}-\sigma_{p_{T}}\right)$ and $\left(\gamma_{T}-\sigma_{\gamma_{T}}\right.$, $\left.p_{T}-\sigma_{p_{T}}\right)$, the corresponding $S_{3}^{\text {ex }}$ are extracted and $\sigma_{S_{3}^{\text {ex }}}$ is taken to be the maximum difference between these four values with the central value $S_{3}^{\text {ex }}$ being calculated with parameters $\gamma_{T}$ and $p_{T}$.

\subsection{Overall $S_{3}$ uncertainty}

Summarising all the studies and results described previously, the $S_{3}$ value inside the cavity can be written by taking into account all the uncertainties of the optical system as:

$$
S_{3}=S_{3}^{\text {elli }}+\delta S_{\text {ex }} \pm \sigma_{S_{3}^{\text {elli }}} \pm \sigma_{S_{3}^{\text {ex }}} \pm \sigma_{\text {time }} \pm \sigma_{\text {trans }}
$$

where $S_{3}^{\text {elli }}$ is the degree of circular polarisation measured using the ellipsometer, $\delta S_{\text {ex }}$ is the $S_{3}^{\text {elli }}$ dependent correction factor defined in Eq.(3.4), $\sigma_{S_{3} \text { elli }}$, of the order of a few $10^{-4}$, is the uncertainty on the measurement of $S_{3}^{\text {elli }}$ using the ellipsometer (Sect. 3.1), $\sigma_{S_{3}^{\text {ex }}}$, of the order of a few $10^{-4}$, is the uncertainty on the determination of the transfer matrix $M_{T}$ (Sect. 3.2.3), $\sigma_{\text {time }} \approx 3 \times 10^{-3}$ is the conservative uncertainty associated to the duration of of data taking of an ellipsometer data sample (Sect. 3.1), and $\sigma_{\text {trans }}<3 \times 10^{-5}$ is the uncertainty related to the passage of the light through the exit cavity mirror (Sect. 3.2.1). The last two uncertainties $\sigma_{\text {time }}$ and $\sigma_{\text {trans }}$ are common to all $S_{3}$ measurements, all others vary for each measurement of $S_{3}$.

\section{Coherence of $S_{3}$ along the whole optical system}

Although the previous studies have provided values of $S_{3}$ at the electron-laser IP with an uncertainty around three per-mill, the idea is now to characterise also the entrance optical elements by a matrix $M_{E}$, determine the values of $S_{3}$ at different places of the optical system to check their coherence and make sure that no additional unknown large effect could induce a bias on $S_{3}$ at the center of the cavity.

\subsection{Determination of $M_{E}$}

The entrance beam line is described with the matrix $M_{E}$ (see Fig. 1) and is composed of a glass plate, two lenses and four alignment mirrors. Following the optical theorem of R. Clark Jones [9] already used in Sect. 3.2.2, $M_{E}$ can be expressed with the same formula (see Eq.(3.2)) as for the matrix $M_{T}$. To determine $M_{E}$, the method pursued is to model the passage of the beam from the entrance Glan polariser to the entrance cavity mirror when the cavity is unlocked, followed by the retro-reflection of the beam by the cavity mirror and its passage through the Glan in the opposite direction. A reversibility theorem [6, 10] states that for a matrix $M$ describing the light path through a given system, the matrix corresponding to the light path in the opposite direction is the transposed matrix of $M$. Following this theorem and starting with a horizontal linear polarisation state $\mathbf{E}_{\text {lin }}=$ $(1,0)^{T}$ just after the entrance Glan, the expression of the retro-reflected field $\mathbf{E}_{\text {ret }}$ and the associated intensity $T_{\text {ret }}$ emerging from the Glan in the return direction can then be written as:

with

$$
\begin{aligned}
& \mathbf{E}_{\mathrm{ret}}=G_{v} M_{\mathrm{QM}}^{T} M_{m} M_{\mathrm{QM}} \mathbf{E}_{\text {lin }}, \quad T_{\text {ret }}=\left|\mathbf{E}_{\text {ret }}\right|^{2} \\
& M_{\mathrm{QM}}=M_{E} R_{\mathrm{QE}} M_{\mathrm{QW}} R_{\phi_{\mathrm{ent}}},
\end{aligned}
$$


where $G_{v}$ is the matrix of the Glan polariser allowing only the vertical component of the field to pass when the beam returns, $M_{\mathrm{QM}}$ is the transfer matrix of the optical line from the plate $\mathrm{QWP}_{\mathrm{ent}}$ to the last alignment mirror, $M_{m}$ is the Jones matrix of the entrance cavity mirror, $M_{E}$ is the transfer matrix to be determined, $M_{\mathrm{QW}}$ is the Jones matrix of the plate $\mathrm{QWP}$ ent , and $R_{\mathrm{QE}}$ and $R_{\phi_{\mathrm{ent}}}$ are two $2 \times 2$ rotation matrices introduced to reflect the azimuthal orientation of $\mathrm{QWP}_{\text {ent }}$ with respect to the matrix $M_{E}$ and to the Glan polariser axes, respectively.

According to this modelisation, the elements of $M_{E}$ have been determined from data recorded with the photodiode $\mathrm{pd}_{\mathrm{ent}}$ for various positions $\phi_{\mathrm{ent}}$ of the plate $\mathrm{QWP}_{\mathrm{ent}}$, by minimising the following $\chi^{2}$ function:

$$
\chi^{2}=\sum_{i=1, N_{\mathrm{ent}}}\left(\frac{R T_{\mathrm{ret}}^{i}-I_{\mathrm{ret}}^{i}}{\sigma_{I_{\mathrm{ret}}^{i}}}\right)^{2}
$$

where $N_{\text {ent }}$ is the number of different azimuthal positions $\phi_{\mathrm{ent}}, T_{\mathrm{ret}}^{i}\left(I_{\mathrm{ret}}^{i}\right)$ is the theoretical (experimental) intensity calculated with Eq.(4.1) (measured with $\mathrm{pd}_{\mathrm{ent}}$ ) at the $i^{\text {th }}$ value of $\phi_{\mathrm{ent}}, \sigma_{I_{\mathrm{ret}}}$ is the uncertainty of $I_{\text {ret }}^{i}$, and $R$ is a normalisation factor which is determined by solving $\partial \chi^{2} / \partial R=0$. When the system was conceived, the characterisation of the entrance optical line was not planned and the photodiode $\mathrm{pd}_{\text {ent }}$ was only devoted to find the azimuthal positions of the plate $\mathrm{QWP}_{\text {ent }}$ leading to a right or left circular polarisation of the laser beam. No specific study has thus been conducted to study the response and measurement uncertainties of this photodiode, and in particular no photodiode thermal regulation and no additional reference photodiode to compensate the laser power variations have been installed. A measurement $I_{\text {ret }}^{i}$ using the photodiode $\mathrm{pd}_{\mathrm{ent}}$ thus corresponds simply to the mean value over ten thousand signals recorded with a 12-bit analog-todigital converter at a sample rate of $2 \mathrm{MHz}$. The uncertainty $\sigma_{I_{\text {ret }}^{i}}$ is defined as the RMS value of the distribution of $I_{\text {ret }}^{i}$ and is of the order of one to two percent. This level of precision is not as good as the one obtained with the ellipsometer photodiodes as described in Sect. 2.1.2, and consequently, the development of a complete theoretical model to describe each optical component would not make sense here. It is therefore sufficient to consider the Glan polariser, the plate $\mathrm{QWP}_{\text {ent }}$ (which is a quartz plate treated with an anti-reflection coating) and the cavity mirror as perfect and to write the corresponding expression of the Jones matrices used in Eq.(4.1) as:

$$
G_{v}=\left(\begin{array}{ll}
0 & 0 \\
0 & 1
\end{array}\right), \quad M_{m}=\left(\begin{array}{ll}
1 & 0 \\
0 & 0
\end{array}\right), \quad M_{\mathrm{QW}}=\left(\begin{array}{cc}
1 & 0 \\
0 & e^{-i \pi / 2}
\end{array}\right) .
$$

A Monte Carlo study shows that our data are well described by using only one delay plate and one polariser. The parameters in Eq.(3.2) are therefore restricted to $\gamma_{1} \equiv \gamma_{E}, p_{1} \equiv p_{E}, p_{2}=1$, $\theta_{3}=0$ and $\gamma_{2}=0$, and the minimisation of the $\chi^{2}$ defined in Eq.(4.2) leads to $\gamma_{E}=(-32.8 \pm$ $0.5) \mathrm{mrad}$ and $p_{E}=1.17 \pm 0.01$. The effect of the matrix $M_{E}$ is clearly visible in Fig. 7 showing the distribution of the ratio $I_{\text {ret }} /\left(R T_{\text {ret }}\right)$ either for the case where the minimisation is performed (dotted line) or for the case where the matrix $M_{E}$ is fixed to the identity (full line).

\subsection{Coherence of $S_{3}$}

Using the matrices $M_{T}$ and $M_{E}$, the $S_{3}$ values at the four different locations $S_{3}^{\text {ent }}, S_{3}^{\mathrm{in}}, S_{3}^{\mathrm{ex}}$ and $S_{3}^{\text {elli }}$ indicated in Fig. 1 can now be determined for any polarisation state of the laser beam, i.e. for any azimuthal position $\phi_{\mathrm{ent}}$ of the motorised rotating mount $\mathrm{QWP}_{\mathrm{ent}}$. These four values are 


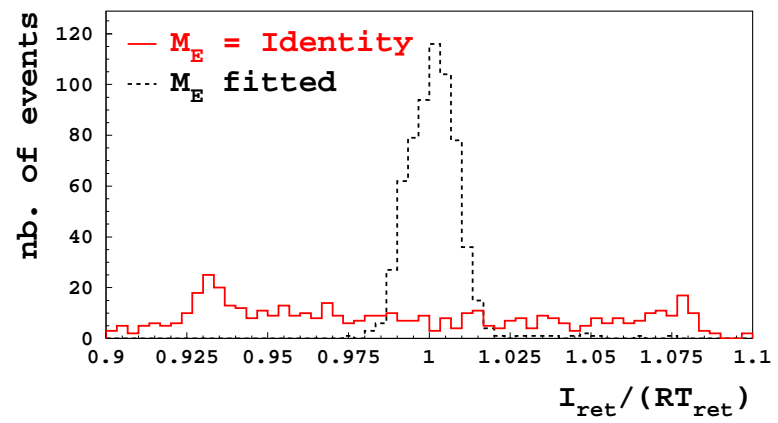

Figure 7. Ratio of the measured intensities over the theoretical ones $I_{\text {ret }} /\left(R T_{\text {ret }}\right)$ comparing the case where the $M_{E}$ is from the fit (dotted line) and the case where the $M_{E}$ is fixed to the identity (full line).

determined from their associated electric fields $\mathbf{E}_{\text {ent }}, \mathbf{E}_{\text {in }}, \mathbf{E}_{\mathrm{ex}}$ and $\mathbf{E}_{\text {elli. }}$. We recall that $\mathbf{E}_{\text {ent }}$ is calculated from a linearly polarised beam passing through the plate $\mathrm{QWP}_{\text {ent }}$ which is positioned at the azimuthal angle $\phi_{\text {ent }}$ with respect to the entrance Glan axis: $\mathbf{E}_{\text {ent }}=M_{Q W} R_{\phi_{e n t}}(1,0)^{T}, \mathbf{E}_{\text {in }}$ is derived from $\mathbf{E}_{\text {ent }}$ and from the matrix $M_{E}: \mathbf{E}_{\text {in }}=M_{E} \mathbf{E}_{\text {ent }}, \mathbf{E}_{\text {elli }}$ is determined as described in Sect. 3.1 from an ellipsometer data sample, and $\mathbf{E}_{\mathrm{ex}}$ is calculated from the field $\mathbf{E}_{\text {elli }}$ and the matrix $M_{T}$ as: $\mathbf{E}_{\mathrm{ex}}=M_{T}^{-1} \mathbf{E}_{\mathrm{elli}}$.

To check experimentally the coherence of $S_{3}$ along the optical system, several arbitrarily values of $\phi_{\text {ent }}$ have been chosen close to a circularly polarised light state and, for each one of these positions, a data sample has been recorded in the ellipsometer as described in Sect. 3.1. The evolution of $S_{3}$ along the optical path can be followed in Fig. 8 through the values of $S_{3}^{\text {ent }}, S_{3}^{\text {in }}$,

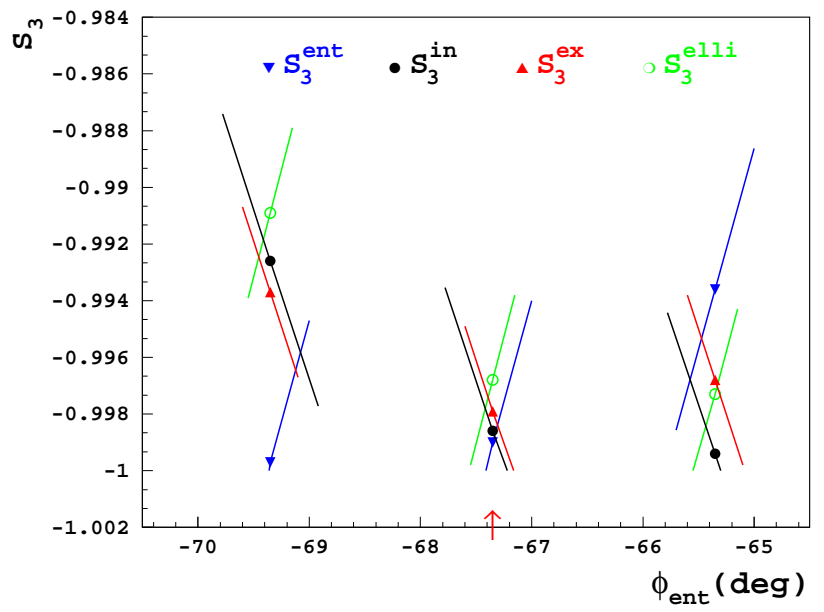

Figure 8. $S_{3}^{\text {ent }}$ (blue triangles), $S_{3}^{\text {in }}$ (black points), $S_{3}^{\text {ex }}$ (red triangles), and $S_{3}^{\text {elli }}$ (open green circles) for three azimuthal positions of the plate $\mathrm{QWP}_{\text {ent }}$ around a left circularly polarised state (indicated by an arrow). The error bars are inclined for clarity. 
$S_{3}^{\mathrm{ex}}$ and $S_{3}^{\text {elli }}$ presented for three positions $\phi_{\mathrm{ent}}$ around a left circularly polarised state. In Fig. 8 , an uncertainty of $0.5 \%$ on the values of $S_{3}^{\text {ent }}$ is taken (typical known value as mentioned in the introduction). This uncertainty propagates directly to that of $S_{3}^{\text {in }}$.

For the measurement of the lepton beam polarisation, the only relevant quantity is the light polarisation inside the cavity, to which, of course, we do not have access but which is located between the two values $S_{3}^{\text {in }}$ and $S_{3}^{\text {ex }}$. As shown in Fig. 8, the difference $\left|S_{3}^{\text {ex }}-S_{3}^{\text {in }}\right|$ is less than one per-mill when $S_{3}^{\text {ex }}$ is closer to -1 (i.e. when the system is at its operating point [1]), and can reach up to three per-mill in the explored domain of $\phi_{\text {ent }}$. Part of the difference could be explained by the presence of a small birefringence due to multi-layers coating cavity mirrors as mentioned in Sect. 3.2.1. We do not know the exact value of our mirror coating birefringence, but birefringences have been measured for instance in [27, 28, 29] for cavity finesses of $6600-100000$. In all these measurements the order of magnitude of the birefringence is a few $10^{-6} \mathrm{rad}$. The Fabry-Perot cavity, with its multi-layer coating mirrors, has a finesse of about 30000 [1] and thus lies within the range quoted above. Because of the resonant optical cavity, the phase shift due to a single passage of the light in the reflected coating is amplified by a factor $2 \mathscr{F} / \pi$ [29] and becomes of the order of a few $10^{-2} \mathrm{rad}$. The bias on $S_{3}$ can be expressed in term of this amplified birefringence $\phi_{\mathrm{bir}}$ as $S_{3}^{\text {ex }}-S_{3}^{\text {in }} \approx \phi_{\text {bir }}^{2} / 2$ [19] and can therefore be of a few per-mill. However, another systematic source, which could explain the difference of a few per-mill between $S_{3}^{\text {in }}$ and $S_{3}^{\text {ex }}$, is the lack of precision in measurements with $\mathrm{pd}_{\mathrm{ent}}$ used for the determination of $M_{E}$ (Sect.4.1) and thus of $S_{3}^{\text {in }}$. Anyway, the study of the entrance beam line does not intend to give an accurate measurement of $S_{3}^{\text {in }}$ but is devoted to check the coherence of the system and particularly the coherence of measurements just before and after the cavity.

\section{Summary}

The implementation of an uncoated QWP in the ellipsometer of the Fabry-Perot cavity polarimeter of HERA has allowed us to determine the degree of circular polarisation $S_{3}$ of the laser at the entrance of the ellipsometer with an uncertainty of $0.3 \%$. Such a small uncertainty is achieved thanks to a complete model description of the ellipsometer optical system. The transport of $S_{3}$ up to the electron-laser IP has then been studied and the modeling of the optical elements located between the IP and the ellipsometer has made it possible to conserve the uncertainty of $0.3 \%$ at the IP. A study of the optical line from the IP up to the laser head has also been performed and has shown that even with an unoptimised photometric measurement, $S_{3}$ is controlled along the optical path at the few per-mill level. The level of accuracy presented here has, to our knowledge, never been reached in the environment of a particle collider and provides a good prospect for applications in a future linear collider [30, 31, 32].

\section{Acknowledgment}

We would like to thank T. Cacérès, M. Delbard, N. Falletto, M. Linz, A. Reboux and M. Woods for their help to this work. 


\section{References}

[1] S. Baudrand et al., A High Precision Fabry-Perot Cavity Polarimeter at HERA, LAL 10-43 [arXiv:1005.2741 [physics.ins-det]].

[2] R.C. King, Thesis, SLAC-REPORT-452 (1994)

[3] A. Lath, Thesis, SLAC-REPORT-454 (1994)

[4] N. Falletto, Thesis Univ. J. Fourier-Grenoble 1, DAPNIA/SPhN-99-03T.

[5] N. Falletto et al., Nucl. Instr. and Meth. A 459 (2001) 412-425.

[6] R. Clark Jones, J. Opt. Soc. Am. 31 (1941) 488.

[7] J. Poirson et al., App. Opt. 34 (1995) 6806-6818.

[8] M. Woods, private communication

[9] R. Clark Jones, J. Opt. Soc. Am. 31 (1941) 493.

[10] N. Vansteenkiste, P. Vignolo, and A. Aspect, J. Opt. Soc. Am. A 10 (1993) 2240-2245.

[11] E.D. Black, Am. J. Phys. 69, (2001) 79-87.

[12] M. Linz, private communication.

[13] Serge Huard, Masson, Paris, 1993.

[14] R. Clark Jones, J. Opt. Soc. Am. 37 (1947) 107-110.

[15] R.M.A. Azzam and N.M. Bashara, Amsterdam, North-Holland, 1997.

[16] F. Zomer, J. Opt. Soc. Am. A 20 (2003) 172-182.

[17] F. Zomer, Opt. Com. 252 (2005) 355-368.

[18] F. Zomer, J. Phys. D: Appl. Phys. 36 (2003) 2697-2704.

[19] M. Jacquet, Habilitation thesis, LAL 09-210 (2009) [http://publication.lal.in2p3.fr/2009/hdr-MJacquet.pdf].

[20] A. Carvallo, Comptes rendus des scéances de l'académie des sciences 126 (1989) 728.

[21] G. Ghosh, Opt. Com. 163 (1999) 95-102.

[22] J.H. Shields and J.W. Ellis, J. Opt. Soc. Am. 46 (1956) 263.

[23] T. Toyoda and M. Yabe, J. Phys. D: App. Phys. 16 (1983) 97-100.

[24] F. Zomer, Habilitation thesis, LAL 03-12 (2003), [http://publication.lal.in2p3.fr/2003/LAL03.12.pdf].

[25] Warren J. Smith, Modern Optical Engineering, McGraw-Hill, 2000.

[26] A. Delbart, DSM/DAPNIA 6-6112 R 2000020 (1999).

[27] D. Jacob et al., Opt. Lett. 20 (1995) 671.

[28] J.Y. Lee et al., Appl. Opt. 39 (2000) 1941.

[29] F. Brandi et al., Appl. Phys. B 65 (1997) 351.

[30] K. Long and K.P. Schüler, Nucl. Instr. and Meth. A 494 (2002) 75-80.

[31] K.P. Schüler, Proceedings of the Linear Collider Workshop 2000, Fermilab, Batavia, IL, USA,

[32] G. Moortgat-Pick et al., Phys. Rep. 460 (2008) 131-243. 